Marquette University

e-Publications@Marquette

Master's Theses (2009 -)

Dissertations, Theses, and Professional Projects

\title{
Towards Predicting Risky Behavior Among Veterans With PTSD by Analyzing Gesture Patterns
}

Tanvir Roushan

Marquette University

\section{Recommended Citation}

Roushan, Tanvir, "Towards Predicting Risky Behavior Among Veterans With PTSD by Analyzing Gesture Patterns" (2018). Master's Theses (2009-). 475.

https://epublications.marquette.edu/theses_open/475 


\section{TOWARDS PREDICTING RISKY BEHAVIOR AMONG VETERANS WITH PTSD BY ANALYZING GESTURE PATTERNS}

by

Tanvir Roushan, B.S.

A thesis submitted to the Faculty of the Graduate School,

Marquette University,

in Partial Fulfillment of the Requirements for

the Degree of Master of Science

Milwaukee, Wisconsin

August 2018 


\title{
ABSTRACT \\ TOWARDS PREDICTING RISKY BEHAVIOR AMONG VETERANS WITH PTSD BY ANALYZING GESTURE PATTERNS
}

\author{
Tanvir Roushan, B.S. \\ Marquette University, 2018
}

\begin{abstract}
Risky behavior including violence and aggression, self-injury, anger outburst, domestic violence along with self-injury, sexual abuse, rule breaking, use of drugs and alcohol, suicide etc. are alarming issues among US military veterans who return from combat zone deployment in Iraq and Afghanistan. Veterans are exposed to trauma in war zones which affect most of them with posttraumatic stress disorder (PTSD) or other metal health problems to some degree. Studies have shown that veterans have much higher rates of PTSD than civilians and are more likely to engage in risky behavior. One of the form of displaying and engaging in risky behaviors is through gestures. We collaborated with veterans and social scientists to find the list of 13 gestures that are often used by veterans engaged in risky behaviors.

In this research work, we have collect accelerometer data from subjects performing the above-mentioned gestures and tried to detect them using machine learning techniques. The thesis describes identifying gesture clusters from the accelerometer coordinate data and development of a predictive model that is able to classify the gestures resulting towards the prediction of risky behaviors among the veterans who suffer from PTSD.
\end{abstract}




\title{
ACKNOWLEDGMENTS
}

\author{
Tanvir Roushan, B.S.
}

I sincerely thank my parents and sister Nazia Roushan for being the source of motivation throughout my life. I am extremely grateful to my advisors Dr. Sheikh Iqbal Ahamed and Dr. Zeno Franco for their guidance, support, ideas and insights throughout this process. I would also like to thank my committee member Dr. Thomas Kaczmarek for providing me the domain knowledge on data analysis and visualization. I am very grateful to Dr. Katinka Hooyer for her input and contribution towards my work.

I cannot thank my friends Jiachen Ma, Nadiyah Johnson and Dr. Osman Gani enough for their assistance on different steps of my research for the past couple of years.

Finally, I would like to acknowledge the contribution of Bill Watson, Nancy Smith Watson and Jim Tasse from University of Wisconsin, Milwaukee. Without them we would not have the data for the research. My gratitude to all the veterans and Dryhootch of America for their help with this project. It has been a great honor to work with them. 
ACKTRACT

1.1 Mental Health Problem in US Military Veterans .................................................... 1

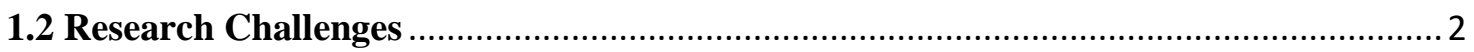

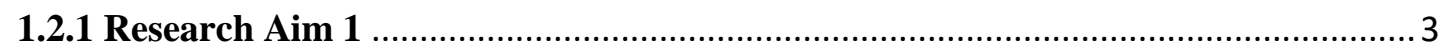

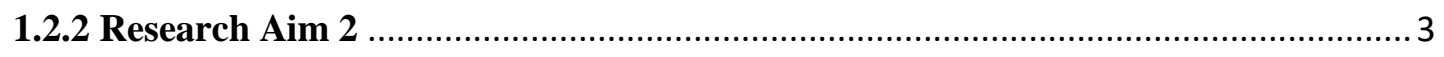

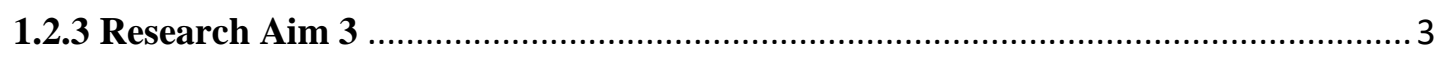

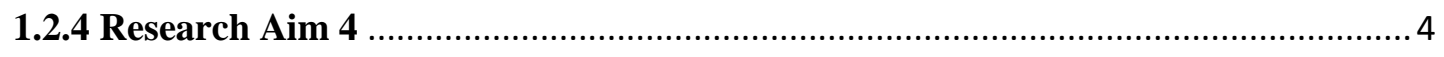

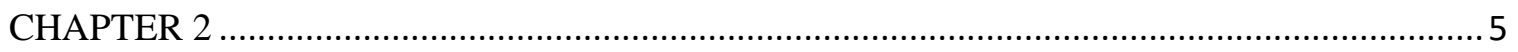

BACKGROUND

2.1 A Review of Risky Behavior Related Research …………………………………....

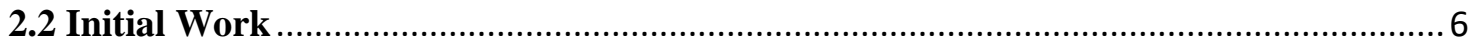

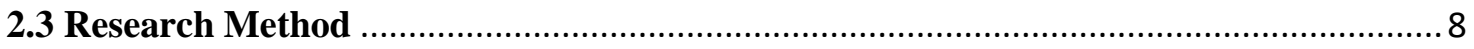

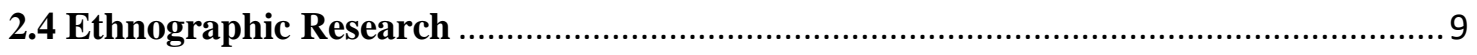


CHAPTER 3

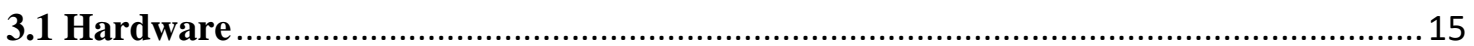

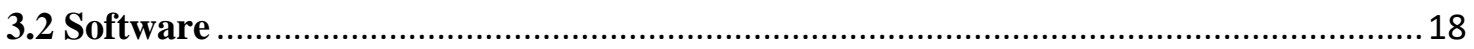

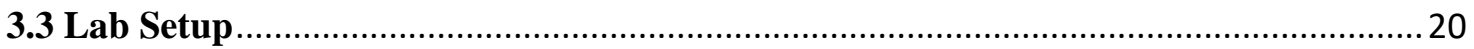

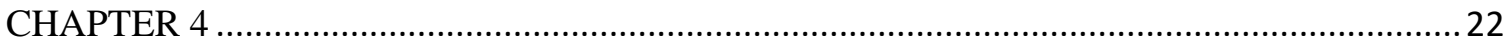

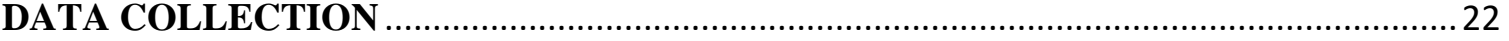

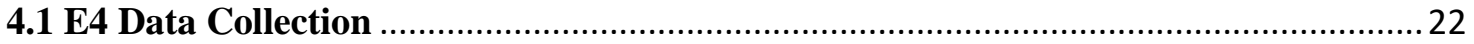

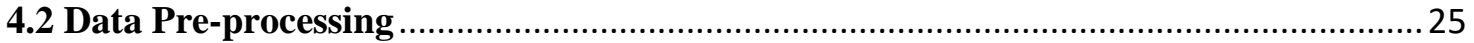

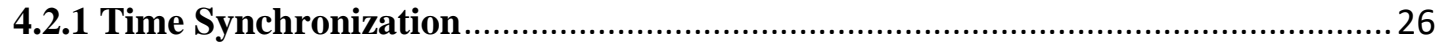

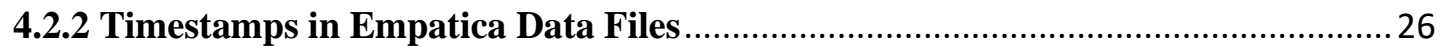

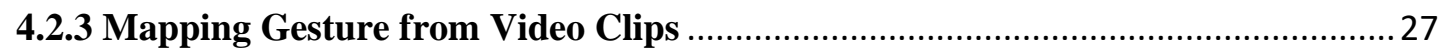

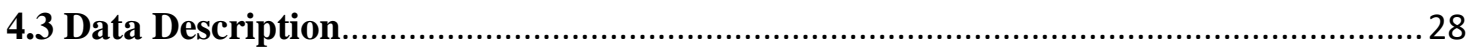

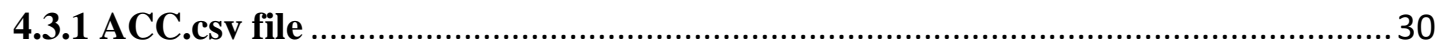

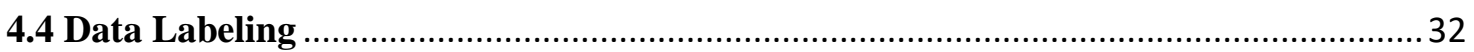

4.4.1 Unix Time to Central Standard Time Conversion ............................................. 32

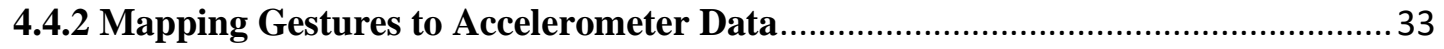

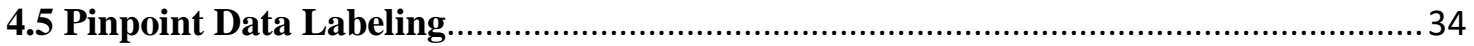

4.5.1 Step 1: Determine Start and End Time of Individual Gesture ..............................35 
4.5.2 Step 2: Label Gestures in Central Standard Time Zone .36

4.5.3 Step 3: Label Accelerometer Data with Gestures

4.6 Data Visualization .39

CHAPTER 5

DATA ANALYSIS

5.1 Expectation Maximization Algorithm 41

5.2 k-Nearest Neighbor Algorithm .43

5.3 Clustering Gesture Data 44

5.4 Classification Algorithms - Supervised Learning 48

5.4.1 Support Vector Machine .50

5.4.2 Distance Weighted k-nearest Neighbors .51

5.4.3 k-nearest Neighbors $(\mathrm{kNN})$ .52

CHAPTER 6 .56

RESULTS .56

6.1 Research Outcome .56

6.2 Distinctively and Reliably Identify Set of Clusters of Aggressive Gestures. .56

6.3 Narrow Down to the Smallest Number of Gesture that are Most Informative .57

6.4 Pin-Point Labeling of the Accelerometer Data and Gestures 57

6.5 Development of a Predictive Model with Classification Algorithms .58 CHAPTER 7 59 


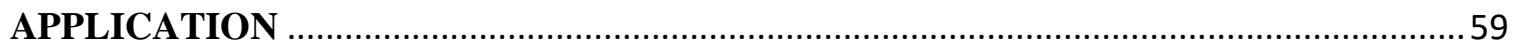

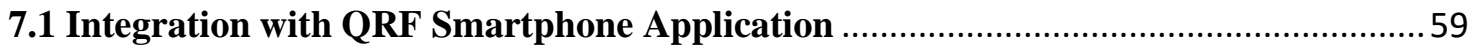

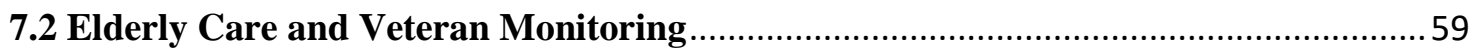

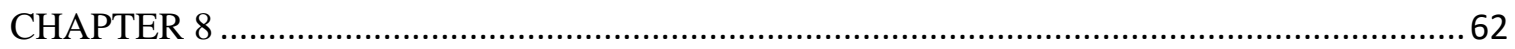

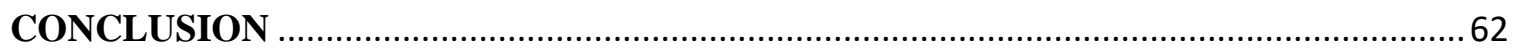

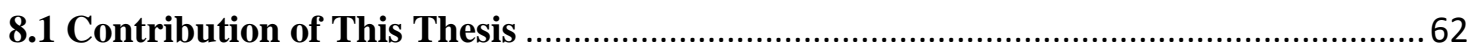

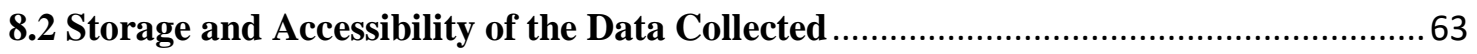

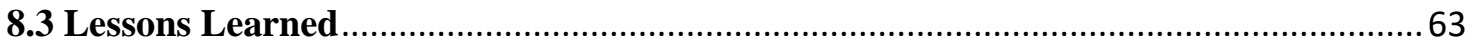

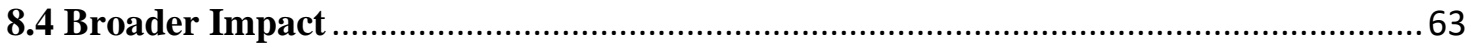

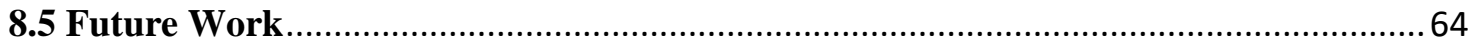

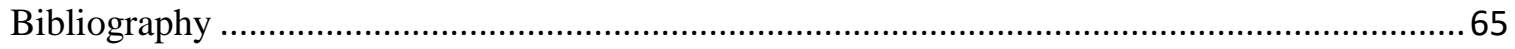

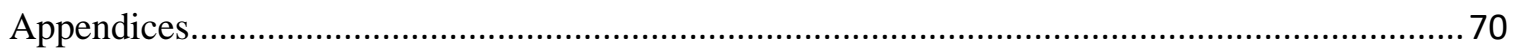

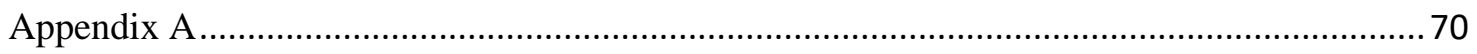

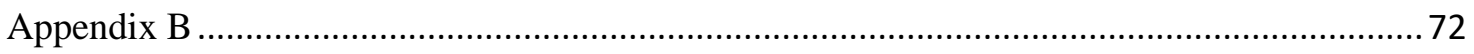




\section{LIST OF TABLES}

Table 2.1 List of Mobile Applications for PTSD .........................................................6

Table 2.2 Low and High Level Aggressive Crisis Behaviors..........................................10

Table 2.3 List of Aggressive Gestures ..................................................................13

Table 3.1 Sensors onboard E4 ..........................................................................

Table 4.1 Summary of E4 sensor data collection .....................................................27

Table 5.1 Top five clusters identified representing the gestures .................................42 


\section{LIST OF FIGURES}

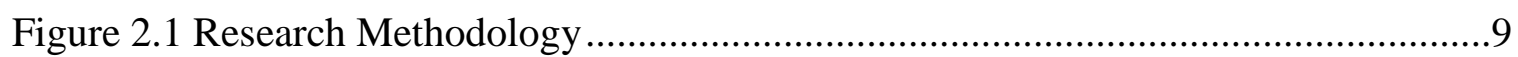

Figure 2.2 Field note from the small story approach in focus group sessions ..................

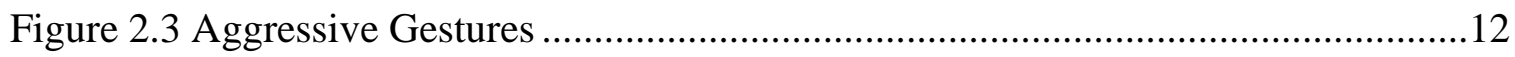

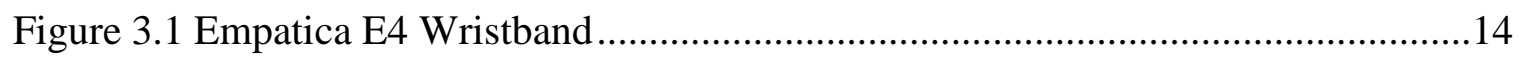

Figure 3.2 Accelerometer sensor data capture model .................................................17

Figure 3.3 Data storage and synchronization process..............................................17

Figure 3.4 E4 Realtime smartphone application for Android phone .............................18

Figure 4.1 Mobile App user interface while live streaming the data to the server ...........21

Figure 4.2 Data collection and Mobile App user interface for streaming the data...........22

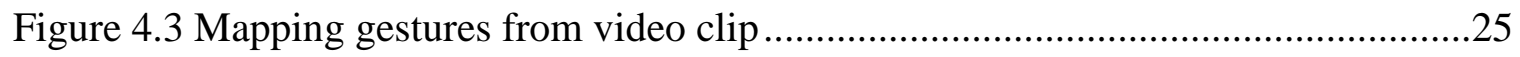

Figure 4.4 General output of the sensors data from Empatica E4 ...............................26

Figure 4.5 The dimensional axes and moving average of ACC …...............................27

Figure 4.6 Raw ACC.csv file from Empatica Connect.............................................28

Figure 4.7 Conversion of Unix Time to CST …...................................................29

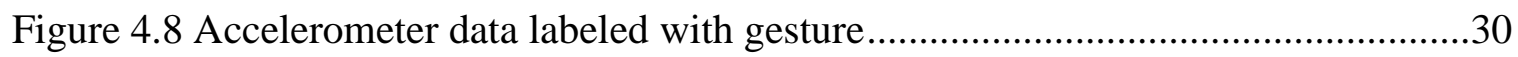

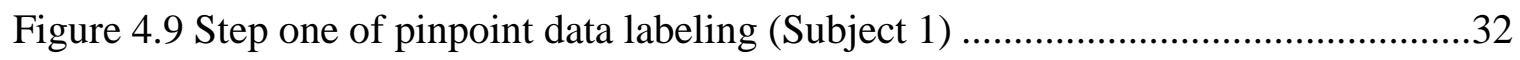

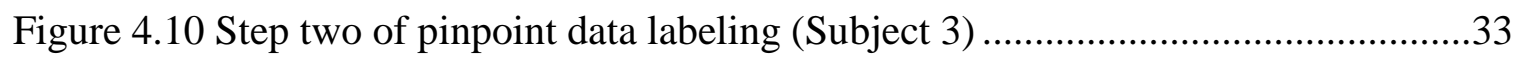

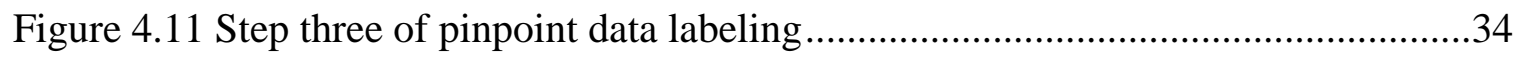

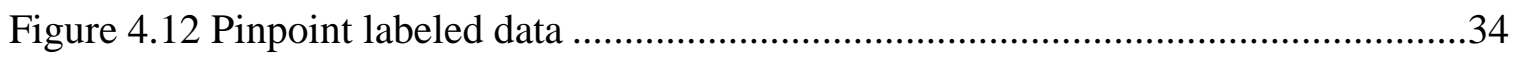

Figure 4.13 Visualization of the labeled accelerometer data form E4 ...........................36

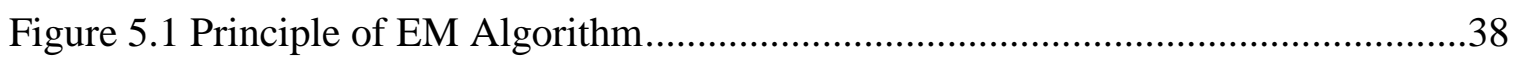

Figure 5.2 (a) Arff viewer in Weka (b) Weka Explorer .............................................40 
Figure 5.3 Output from EM algorithm showing clusters of aggressive gestures .41

Figure 5.4 Output from EM algorithm in Weka

Figure 5.5 Gesture clusters output form EM algorithm. Participant 2..........................43

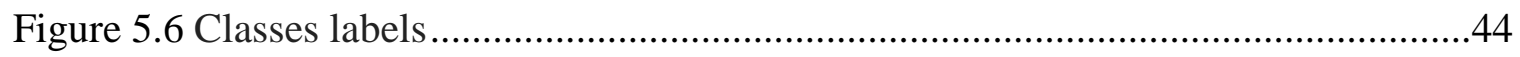

Figure 5.7 Scatter plot of accelerometer sensor data along different axes .....................44

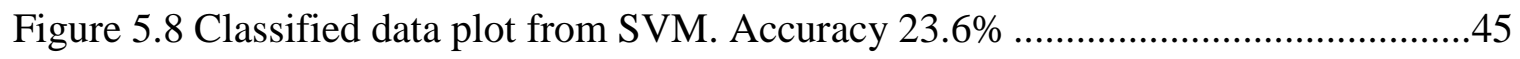

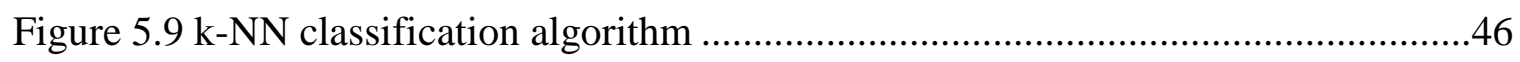

Figure 5.10 (a) Correctly classified data points. (b) Incorrectly classified data points .....47

Figure 5.11 Correctly classified data points using k-NN algorithm. Accuracy $66.7 \%$....47

Figure 5.12 Classified data of 12 gestures plotted separately .....................................48

Figure 5.13 Confusion Matrix and ROC Curve .....................................................49

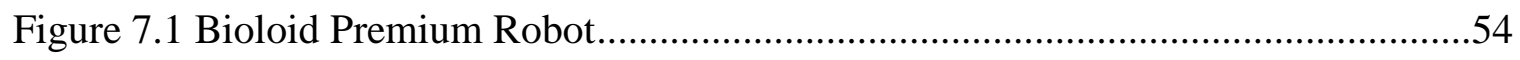




\section{CHAPTER 1}

\section{INTRODUCTION}

\subsection{Mental Health Problem in US Military Veterans}

One of the worrying issues among US military veterans who return from deployment in combat zones in Iraq and Afghanistan are mental illness from trauma. Often, they suffer mental health issues like post-traumatic stress disorder (PTSD) from the ordeal of combat. Many veterans experience troubles reintegrating back to society and civilian life style once they return home. That results in ending up being jobless, loss of home, domestic issues, drug problems etc. These issues slowly push the veterans towards being isolated from family, friends and society. As a consequence, they endure more mental stress and engage in risky behavior and engage in crisis situations. Anxiety, self-injury, anger outburst, aggressive behavior, domestic violence, rule breaking, sexual violence, suicide, use of alcohol and drugs are disturbing issues that are included among the risky behavior. Countless Iraq and Afghanistan military veterans are exposed to immense trauma that affects them to PTSD or other mental health problems to some degree.

There are studies that shown that veterans who are exposed to more depressive and PTSD symptoms are reported to express more risky behaviors. [1]. Acting recklessly is a symptom of disorder and risky behavior. Reckless behavior can increase and, in some cases, worsen the PTDS among veterans. Is indicates when veterans are involved in risky behavior they end up increasing the odds of another traumatic experience. Veteran population have a higher rate of PTSD compared to the civilian population. They are more likely to engage in risky behavior. PTSD affects 1 in 10 Afghanistan veterans and 1 
in 5 Iraq veterans. The suicide risk among veterans are $50 \%$ higher than that of the civilian population according to the United States Department of Veteran Affairs (VA) [2]. The VA has taken initiatives over the years to tackle the mental health (MH) issues among the veterans. However, the issue is still at large and generating more attention day by day. The VA's National Center for PTSD recommends re-conceptualizing PTSD [3]. MH problems are also related to early mortality caused from diabetes and cardiovascular diseases.

Risky behaviors lead to crisis events, and by crisis events we mean a situation where a person's behavior make them prone to self-injury or hurting others. Our research aims to detect the risky behavior among veterans by studying and analyzing their gesture pattern. To identify the early warning signs of risky behaviors, we focused on determining the gestures by conducting an extensive ethnographic study. In this research we have collected accelerometer data and physiological data from wearable sensors and looked into the accelerometer data to identify gestures using computational approach including machine learning and data analysis. This thesis paper describes our approach to identify gesture clusters from the accelerometer coordinate data and development of a predictive model that is able to classify the gestures. The outcome of the thesis results towards the prediction of risky behaviors among the veterans who suffer from PTSD. By predicting risky behavior, we intend to intervene if possible before a crisis event goes out of hand.

\subsection{Research Challenges}

The key element to predict a high-risk behavior among the $\mathrm{MH}$ patients (i.e. veterans) is to get timely information and accurate data about these behaviors and 
symptoms. To get timely data we took the approach to collect data from sensors and networks in Internet of Things (IoT). We selected a wearable wristband that collected physiological data and accelerometer data which is connected to the internet via smartphones. We focused entirely on the accelerometer sensor data in this thesis paper. The research aims of the thesis are described below.

\subsubsection{Research Aim 1}

We had collected nearly thirty-nine thousand data points from the accelerometer sensor of the wearable device. Our first research aim is to distinctively and reliably identify a set of clusters from the accelerometer sensor data that represent gestures of aggressive behavior shown by the at-risk veterans who are suffering from PTSD. We intend to use clustering algorithm that is unsupervised machine learning technique to identify the gestures.

\subsubsection{Research Aim 2}

The second goal of this research work is to reduce the number of clusters that we found from output. We wanted to identify the strong gesture clusters that are most informative, in terms of the precision or accuracy, among several gestures detected.

\subsubsection{Research Aim 3}

Reasonable results from any machine learning technique depends largely on the quality of the data that is being used to train the algorithm. Qualitative, accurate and clean training data set increases the chance of a better outcome. The accelerometer sensor data that we collected were solely of an identified set of gesture that the high-risk veterans make. Over thirty-eight thousand data points were collected and labeled with respective 138 gestures for this purpose. However, the labeling was not efficient enough 
to accurately label 129 gestures. The third research challenge was to pin-point label the data we collected.

\subsubsection{Research Aim 4}

The fourth research aim of this research was to develop a predictive model that could detect the gestures from the pin-point labeled data set. We intended used several supervised machine learning approaches to classify the gestures. Our aim was to train a system well enough so that the model would be able to classify the member class (i.e. gestures) from any given accelerometer sensor reading data set. 


\section{CHAPTER 2}

\section{BACKGROUND}

\subsection{A Review of Risky Behavior Related Research}

A number of research efforts have focused on detecting gestures from sensors data, analyzing gait patterns and image recognition. Equally there is evidence of research on human behavior and risky behavior detection. Numerous researches are conducted to detect behavior and emotion automatically that are presented in [4, 5-7]. While there are huge number of studies done on emotion recognition from one source of data, human behavior experts suggest that information seen from a single source of data is often not enough to identify emotions. [8, 9]. A user-dependent system uWave [10] was developed that can recognize eight simple gestures with an accuracy of $98.6 \%$. Another system iCap was developed [11] that is used to comprehend the mental model of it users based on context awareness. In our research we aim to merge these two fields of behavior and gesture recognition together. To our knowledge, there is no existing dataset of gestures related specifically to aggression outcome. We have focused on identifying gestures and predict risky behavior from accelerometer data.

Even if we can predict crisis events and signs of risky behavior by analyzing the gestures of the veterans, we need to deliver that information to a family member, a peer support provider, or a clinician who can intervene and act. A convenient way to send this time critical information to others is to use a smartphone application. The VA lists a number of phone applications that are ready to use by the veterans. These applications include self-help apps and treatment companion apps to support veterans going through trauma [12]. Table 2.1 lists the smart phone applications provided from the VA. 


\begin{tabular}{|l|l|}
\hline \multicolumn{1}{|c|}{$\begin{array}{c}\text { Smartphone } \\
\text { Application }\end{array}$} & \multicolumn{1}{c|}{ Functionality } \\
\hline PTSD Coach & $\begin{array}{l}\text { A self-help application that helps veterans to learn and cope } \\
\text { with PTSD symptoms }\end{array}$ \\
\hline PTSD Family Coach & $\begin{array}{l}\text { This application helps family members of the veterans who } \\
\text { are suffering from PTSD }\end{array}$ \\
\hline Mindfulness Coach & $\begin{array}{l}\text { The application is used by veterans to cope with unpleasant } \\
\text { thoughts and emotions }\end{array}$ \\
\hline VetChange & $\begin{array}{l}\text { Veterans who are suffering from drinking issues are offered } \\
\text { to use this application }\end{array}$ \\
\hline CPT Coach & $\begin{array}{l}\text { This is an application under the treatment companion label. } \\
\text { It allows veterans to work with their therapist }\end{array}$ \\
\hline PE Coach & $\begin{array}{l}\text { Veterans going through Prolonged Exposure therapy are } \\
\text { encouraged to use this application }\end{array}$ \\
\hline CBT-i Coach & $\begin{array}{l}\text { This application is designed to help veterans who suffer } \\
\text { from insomnia }\end{array}$ \\
\hline
\end{tabular}

Table 2.1 List of Mobile Applications for PTSD

\subsection{Initial Work}

Other researchers in our research lab have worked with different aspects of veterans' mental health issues for over 5 years. A study was done on developing a mobile application that aims to assist veterans to reintegrate into civilian life [13]. The continuation of that work was conducted in two other research that developed applications that coordinate data collection that are potentially relevant for monitoring high-risk behaviors in veterans [14] and a generic m-health tool for continuous assessment, automatic intervention and analysis of veteran mental health related issues 
[15]. Another recent study explored the area of developing a social-context middleware for at-risk veterans [16]. The middleware solution is a system that detect anger outburst and possibly prevent it by taking social context aware technology. The research took wearable sensor data and global positioning system (GPS) data into account to detect risky behavior of veterans and intervene in a timely manner.

The research team worked closely with the Dryhootch of America, a non-profit organization that is formed by combat veterans to help veterans in their return home [17]. The partnership with Dryhootch enabled us to be involved directly with community engagement. The partnership has produced several research products linked to community-based veteran engagement in $\mathrm{MH}$ and general health care with veterans. As a part of the research [15] a peer-mentor support smartphone application Quick Reaction Force (QRF) was developed. Throughout the development process the research team thoroughly integrated feedback from the veterans. QRF had two parts, one used by the peers (at-risk veterans) and the other one is used by the mentors (veterans trained to deal with at-risk veterans). The application is used as a platform to collect prototype selfreport data from veterans and assessment data about the veteran from a peer mentor. Weekly surveys are generated and sent to at-risk veterans suffering from PTSD. The survey results are sent to the peer-mentor for reviewed. Based on the response from the veteran a peer-mentor can contact the veteran and intervene if necessary through message or a phone call through the QRF application. All these prior research work in the lab has set the ground towards the research and development of a system that would provide an acceptable prediction of aggressive behavior among veterans with PTSD from smartphone application and sensor data. The ultimate goal is to develop an alert based 
mental health care intervention system that would alert family members and authorities before a veteran engages in a crisis event.

\subsection{Research Method}

The steps of this thesis research methodology have four steps. The steps are illustrated in the flow diagram in figure 2.1. In step 1, we collect 3-axis accelerometer sensor data from the E4 device. In step 2, we label the data with timestamps and gestures. This step includes synchronizing and mapping the time stored in the E4 with the time when gestures were performed by the subjects. In step 3, we ran expectation maximization clustering algorithms on the data we collected to find data clusters that indicate are gestures. Once the clusters are found, we ran supervised learning algorithm k-nearest neighbor on the labeled data to classify them into gestures. We used MATLAB and Weka machine learning tool kit to run the clustering and classification techniques. Finally, in step 4, we evaluated and validated the predictive model from the classification output. 


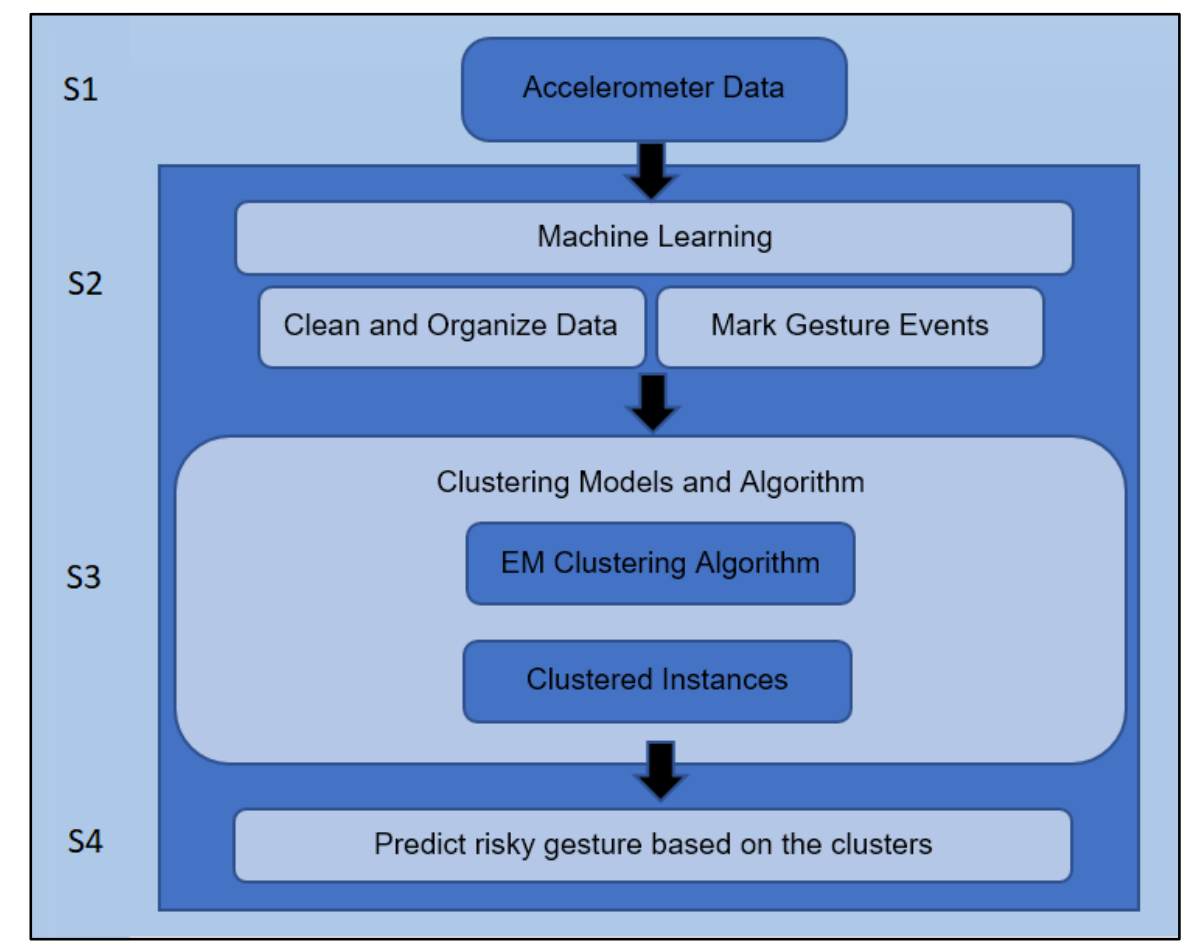

Figure 2.1: Research Methodology

\subsection{Ethnographic Research}

The social scientists in the research team conducted extensive ethnographic research from small focus groups with high risk veterans to observe "lived experience" of veterans with history of anger issues. The study of gesture is an integral part of research in the field of psycholinguistics, psychology, and ethnology among many other fields of social science and anthropology [18]. To understand human behavior and why an individual responds and reacts to a certain situation, studying and understanding their gestures is an important part of this research. In the summer of 2016, the multidisciplinary research team consisting of anthropologist, computer scientist, and clinical psychologists met with three high-risk veterans for seven sessions to conduct the ethnographic research. 


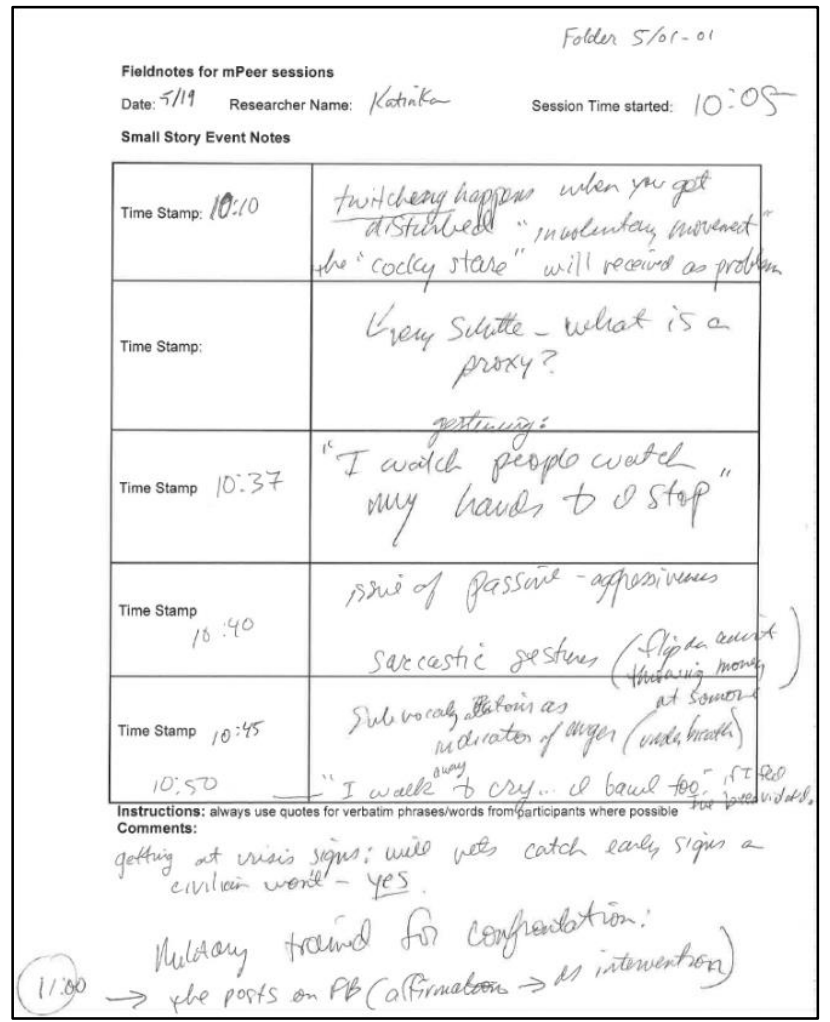

Figure 2.2: Field note from the small story approach in focus group sessions

The social scientists used open-ended interviews to elicit the contexts and bodily experiences leading up to angry outbursts. The weekly focus group sessions lasted from 45 to 60 minutes where the study team used "small story" [19] field note worksheets to document warning signs, triggers and social contexts of anger with these veterans. A sample of the field notes taken during the focus group meetings is shown in the figure 2.2 above.

From the field notes, the anthropologist on our team used descriptive coding to develop a list of dimensions to design a general taxonomy of warning signs (scenarios, social settings, and gestures). This taxonomy was further developed in detail by the entire team by reviewing and commenting on the fieldnotes. We classified the various types of 
cues leading up to an angry outburst in each of the general categories, both social (external) and individual (internal). The detail on the findings from these data can be found in [20]. The findings from the ethnographic research were reduced and this can be seen in table 2.2. This table consists of the aggressive crisis behaviors and gestures the veterans make which are taken from [20].

\begin{tabular}{|c|c|c|}
\hline \multicolumn{3}{|c|}{ Low and High Level Aggressive Crisis Behaviors } \\
\hline Throwing gestures & $\begin{array}{l}\text { - } \text { Money on bar } \\
\text { - Keys on table }\end{array}$ & - Phone across table \\
\hline Hand Gesture & $\begin{array}{l}\text { - Knife hand } \\
\text { - Pointing }\end{array}$ & $\begin{array}{ll}\text { - } & \text { Middle finger } \\
\text { - } & \text { Making a fist/fist in air }\end{array}$ \\
\hline Foot gestures & $\begin{array}{ll}\text { - } & \text { Tapping } \\
\text { - } & \text { Shaking leg }\end{array}$ & - Kicking object \\
\hline Physical contact & $\begin{array}{l}\text { - } \text { Poke } \\
\text { - } \text { Push } \\
\end{array}$ & - Enter other's personal space \\
\hline Melee & $\begin{array}{ll}\text { - } & \text { Throwing bottle } \\
\text { - } & \text { Throwing bar stool }\end{array}$ & $\begin{array}{l}\text { - } \quad \text { Throwing people } \\
\text { - } \quad \text { Chasing people }\end{array}$ \\
\hline Grand gestures & $\begin{array}{ll}\text { - } & \text { Sweep objects off table } \\
\text { - } & \text { Flipping table over }\end{array}$ & - $\quad$ Throwing lamp \\
\hline
\end{tabular}

Table 2.2: Low and High Level Aggressive Crisis Behaviors

\subsection{Simulated Aggressive Gestures}

At this stage we worked with three professional actors Bill Watson, Nancy Smith Watson and Jim Tassem, part of the study team who assisted with embodying what we found from the veteran focus groups. Bill and Jim are actors and directors currently teaching in Peck School of the Arts of University of Wisconsin, Milwaukee. They helped us to simulate rage, frustration, aggressive gestures and behavior and other high 
emotional intensity events. The rationale behind choosing actors rather than any student or volunteer from the lab is these artists are professionally trained to method acting and imbue emotions. The physiological data was precise and the closest to the real-world data that we could collect. The actors acted out the gestures in a series of simulations wearing the E4 wristband in their hand, while we collected data. One actor wore the E4 Empatica device, while another actor made provocative statements for the first actor to respond to. The set of images below in figure 2.3 are a sample set of the gestures the method actors made during the data collection sessions. Top row from left to right: shove, making a fist, knife hand, pointing. Middle row from left to right: Italian street talk. Bottom row from left to right: giving the middle finger, sweeping things off the table, throwing money on bar, pounding fist on a table [21]

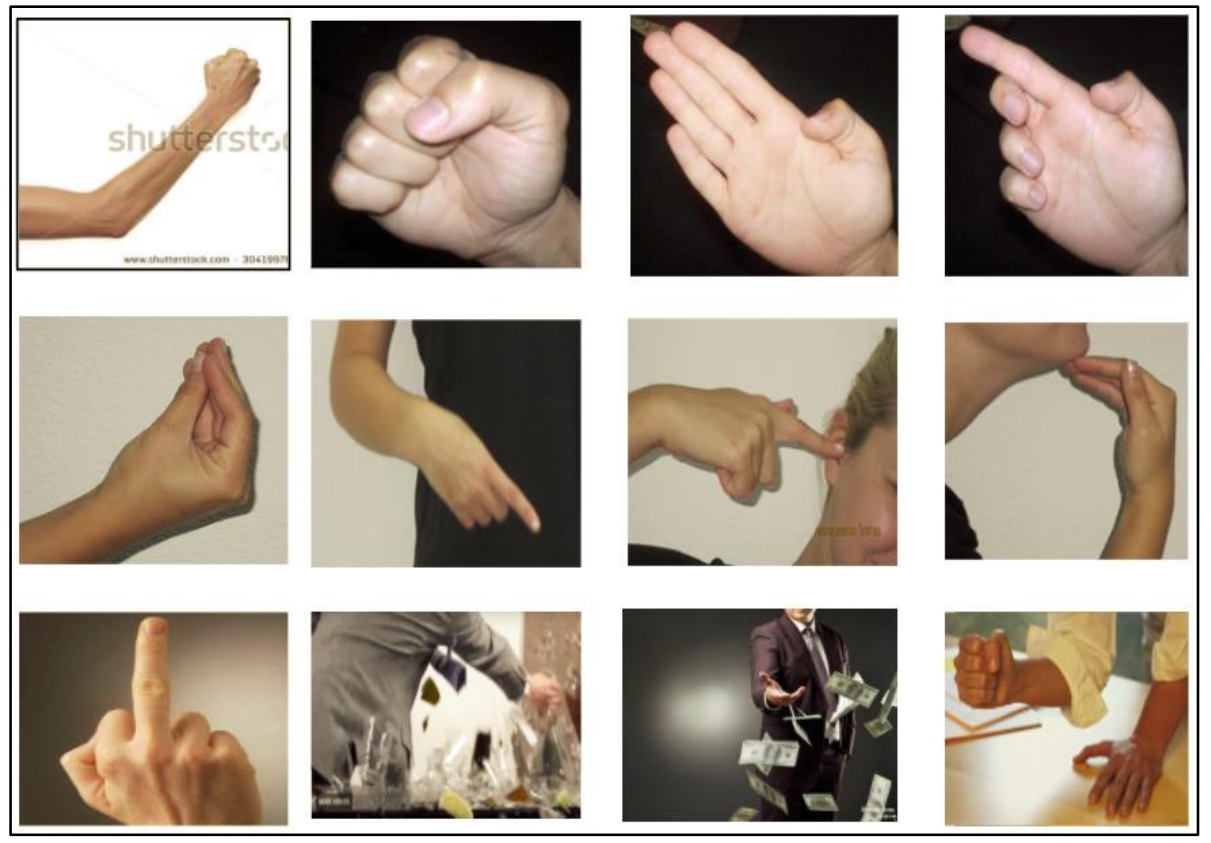

Figure 2.3: Aggressive Gestures 
The social scientists in the research team selected 13 gestures for the actors to act out. They are listed in table 2.3. These gestures were selected based on the findings from the ethnographic study. Each gesture from table 2.3 was performed repeatedly at least for three times so that we could get richer data set and also to make sure the E4 data conform more closely to real-life. The scenarios acted were a formal scripted interaction using Shakespeare and modern theater scripts. The performance session of each actor continued for 12 to 15 minutes. These scenarios involved one high intensity anger condition, one graduated anger condition, and one non-anger but intense emotion condition. During both anger conditions a third actor prompted the two acting out the script to systematically incorporate all anger related gestures if any were missed. However, the actors were also free to use other gestures and were encouraged to improvise [20]. Veterans were present in the room while data was collected. The justification of them being in the room is to ensure member checking for the qualitative research $[22,23]$. They provided credibility and validation of the approach. They gave us important feedback throughout the process.

\begin{tabular}{|l|l|}
\hline Sl. No. & \multicolumn{1}{|c|}{ Gestures } \\
\hline 1 & Fist in the air \\
\hline 2 & Giving the finger \\
\hline 3 & Hands on head \\
\hline 4 & Italian street talk \\
\hline 5 & Knife hand \\
\hline 6 & Pointing \\
\hline
\end{tabular}




\begin{tabular}{|l|l|}
\hline 7 & Poking chest \\
\hline 8 & Pounding fist \\
\hline 9 & Shove \\
\hline 10 & Sweep things off the table \\
\hline 11 & Throwing keys \\
\hline 12 & Throwing things \\
\hline 13 & Throwing money on \\
\hline
\end{tabular}

Table 2.3: List of Aggressive Gestures

The research aims states our goal is to identify clusters from accelerometer sensor data that represents aggressive gestures. The ethnographic research allowed us to determine which gestures veterans are most likely to display in a probable scenario of crisis event. Additionally, the aggressive gesture simulation with the help of the actors explains how we approached to collect the gesture data and the rationale behind using actors rather than real veterans for this research. The next step was to label the data we collected. From the labeled data we would try to apply classification algorithms to develop a predictive model that would accurately classify the gestures. 


\section{CHAPTER 3}

\section{LAB ENVIRONMENT SETUP}

\subsection{Hardware}

We selected the E4 wristband from Empatica for data collection. This is a wearable device used in many areas in clinical trials and in medical research. The wearable device is equipped with a collection of sensors collecting physiological data in real time [24]. Using the E4 device is easy and convenient. Users can wear it like a watch on their wrist. If need be, it can be strapped around the leg as well. Figure 3.1 show the E4 wristband. Data recording starts once the button on E4 is pressed. We selected the E4 device for its unique features. It has an internal memory that stores data for up to 60 hours of observations. The battery provides backup of $48+$ hours of data recording. The full charging time is less than 2 hours. The temporal resolution is 0.2 seconds in streaming mode that gives us the high precision of data measurement with respect to time. Collectively, these features made the E4 the best device selection for this application. Given low frustration tolerance of the veteran population we are interested in ultimately working with using sensors, the E4's unobtrusive ease of use was also a key factor in device selection.

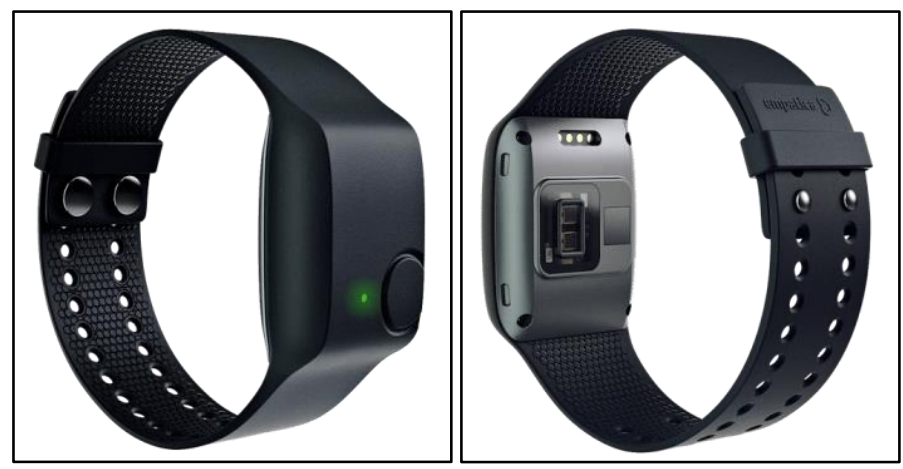

Figure 3.1: Empatica E4 Wristband 
We were able to collect high-quality data with the 5 sensors onboard E4 that provides 6 parameters including 1) accelerometer data, 2) blood pressure volume, 3) heart rate variance, 4) body temperature, and 5) galvanic skin response. The details on the sensors and data unit can be found in Appendix A. The device can be connected to a smartphone over Bluetooth to stream and visualize data in real-time. The state of the art onboard Micro Electro Mechanical Systems (MEMS) sensor chip collects 3-axis accelerometer data (X, Y and $\mathrm{Z}$ axis). The sensor measures continuous gravitational force. For purpose of measurement the International System of Units (SI) denotes the gravitational force of the earth by $g$. The value of $g$ is $9.80665 \mathrm{~m} / \mathrm{s}^{2}[25,26]$. We have rounded up the value to $9.81 \mathrm{~m} / \mathrm{s}^{2}$ for this research. The range is limited to negative $2 \mathrm{~g}$ to positive $2 \mathrm{~g}$. The $\mathrm{E} 4$ can be extended to $+/-8 \mathrm{~g}$ with custom firmware. E4 can capture 32 readings every second. The table 3.1 below lists the sensors and the parameters that are captured using E4.

\begin{tabular}{|l|l|l|l|}
\hline S1. No. & Sensor & Captured parameters & Data and Unit \\
\hline 1 & $\begin{array}{l}\text { 3-axis Accelerometer } \\
\text { X, Y, and Z axis. }\end{array}$ & Captures motion-based activity. & $\begin{array}{l}1 / 64 \mathrm{~g} . \\
\text { Range [-2g, 2g] }\end{array}$ \\
\hline 2 & $\begin{array}{l}\text { Photoplethysmogram } \\
\text { (PPG) Sensor }\end{array}$ & $\begin{array}{l}\text { Measures Blood Volume Pulse } \\
(\mathrm{BVP}), \text { from which heart rate } \\
\text { variability can be derived }\end{array}$ & $\begin{array}{l}\text { Data from } \\
\text { photoplethysmograph }\end{array}$ \\
\cline { 3 - 4 } & $\begin{array}{l}\text { Measures heart Beats Per Minute } \\
(\mathrm{BPM}) \text { from each cardiac cycle }\end{array}$ & $\begin{array}{l}\text { Positive integer. } \\
\text { Numeric count per } \\
\text { minute }\end{array}$ \\
\cline { 3 - 4 } & & Heart rate variance & Seconds (s). \\
\hline
\end{tabular}




\begin{tabular}{|l|l|l|l|}
\hline 3 & $\begin{array}{l}\text { Electrodermal activity } \\
\text { (EDA) Sensor, } \\
\text { Galvanic Skin } \\
\text { Response Sensor } \\
\text { (GSR) Sensor }\end{array}$ & $\begin{array}{l}\text { Measures the constantly fluctuating } \\
\text { changes in certain electrical } \\
\text { properties of the skin }\end{array}$ & Micro-siemens $\mu \Omega$ \\
\hline 4 & Infrared Thermopile & Reads peripheral skin temperature & ${ }^{\circ}$ Celsius \\
\hline 5 & $\begin{array}{l}\text { Internal Real-Time } \\
\text { Clock }\end{array}$ & $\begin{array}{l}\text { Temporal resolution of } 0.2 \text { seconds in } \\
\text { streaming mode }\end{array}$ & Timestamp in UTC \\
\hline 6 & Event Mark Button & $\begin{array}{l}\text { Tags events and link them to } \\
\text { physiological signals }\end{array}$ & $\begin{array}{l}\text { Unix timestamp in } \\
\text { UTC }\end{array}$ \\
\hline
\end{tabular}

Table 3.1: Sensors onboard E4

To understand how the accelerometer sensor unit works to capture data, we can consider the sensor as a cubic box with a ball in the center [27]. If there is no gravitational force working on the cube (i.e. the accelerometer sensor), the ball floats in the center. Figure 3.2 helps us to picture the cube (figure 3.2-a). It has positive x-axis $(\mathrm{X}+)$ and negative $\mathrm{X}$-axis $(-\mathrm{X})$ along the horizontal plane, positive $\mathrm{z}$-axis $(\mathrm{Z}+)$ and negative $\mathrm{z}$-axis $(-\mathrm{Z})$ along the vertical plane, positive $\mathrm{y}$-axis $(\mathrm{Y}+)$ and negative $\mathrm{y}$-axis (Y) going through the plane inside the surface of the page from the reader's perspective. The walls of the cubic spaces are sensitive to pressure. When the cube is moved to the left (figure 3.2-b) with an acceleration of $1 \mathrm{~g}\left(\mathrm{~g}=9.81 \mathrm{~m} / \mathrm{s}^{2}\right)$ the ball hits the right wall $-\mathrm{X}$ on the right with a value of $-1 \mathrm{~g}$ along the $\mathrm{x}$-axis. Let us consider a comparable case where the box is moved up along the positive $\mathrm{z}$-axis (figure 3.2-c) with an acceleration of $1 \mathrm{~g}$. In this case, the ball in the cube would hit the $-\mathrm{Z}$ face of the cube. The pressure sensitive wall records the $\mathrm{g}$ force accordingly. Similarly, the accelerometer sensor captures the $\mathrm{g}$ 
force along all axes. When the cube is moved in a direction along more than one axis (figure 3.2-d) $+X$ and $+Z$ for example, the ball impacts on the opposite walls $(-X$ and $-Z$ ). Although the model described here is not exactly how the MEMS sensors operate, but the principle is the same.

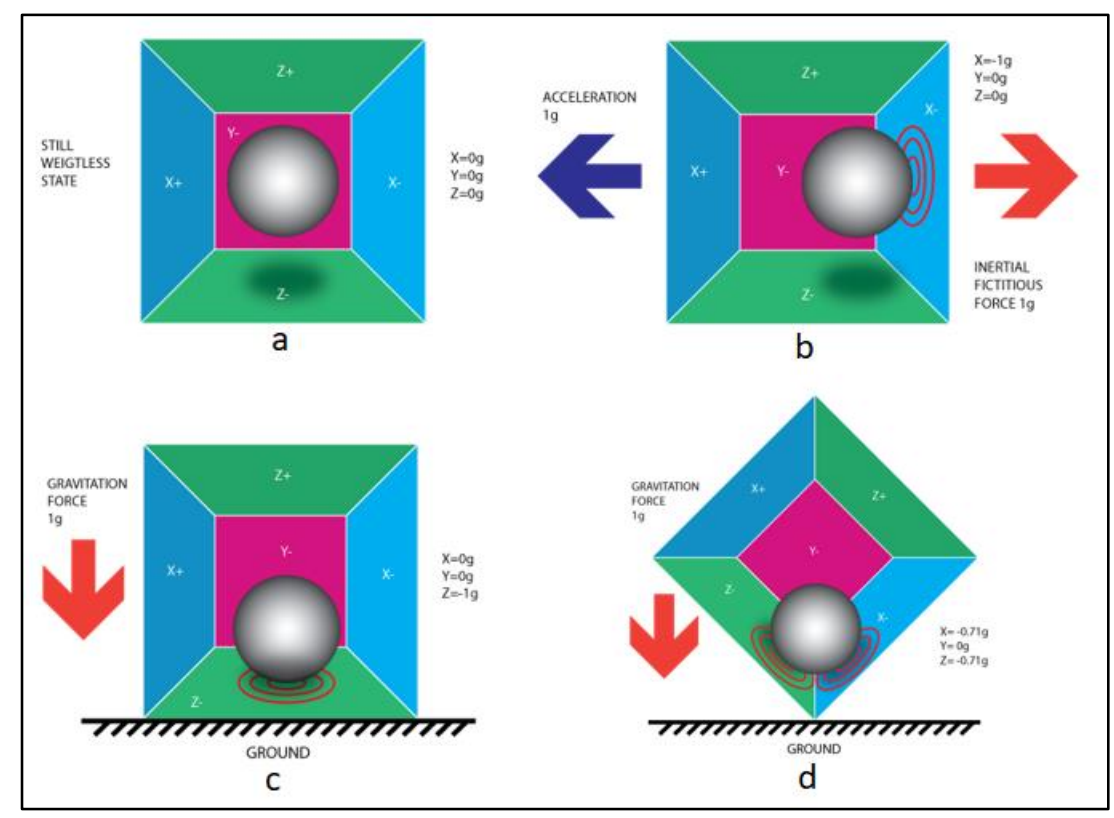

Figure 3.2: Accelerometer sensor data capture model

\subsection{Software}

There are two software components for the E4 wristwatch, a) a mobile application- E4 Realtime and b) a web application- Empatica Connect. User accounts were created online at the empatica website (https://www.empatica.com/connect) before we started collecting the data. Figure 3.3 shows the whole process of data storage from the E4 device to the cloud. In the diagram below from left to the right we can see in step 1, E4 device is collecting data. In step 2, Data is being sent to the mobile application E4 
Realtime. Then in step 3, the smartphone is connected to the Wi-Fi and the data is being pushed to the cloud server. Finally, in step 4, users can login to the web application to further analyze the data from any location.

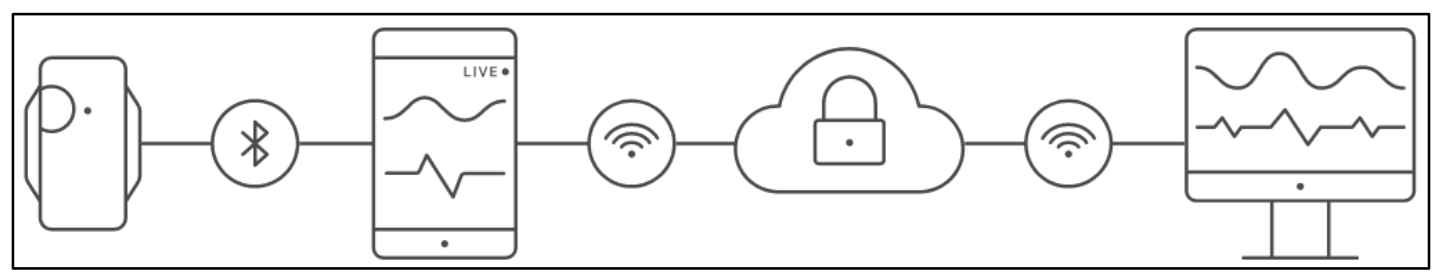

Figure 3.3: Data storage and synchronization process

Empatica comes with a mobile application E4 Realtime to visualize the data in real-time for both Android and iOS platform. Researchers can develop their custom mobile applications by using the API and Android software development kit (SDK) [28]. The E4 stores all the data in its internal memory. As mentioned earlier, data is streamed and sent to the smartphone application E4 Realtime over Bluetooth. When the mobile application is connected to the Wi-Fi data, the data is sent and synchronized to the cloud server. Screenshots from the smartphone application E4 Realtime is shown in Figure 3.4. The application can be downloaded from Apple Store and Google Play. The links are https://itunes.apple.com/us/app/empatica-e4-realtime/id702791633 and https://play.google.com/store/apps/details?id=com.empatica.e4realtime respectively. 


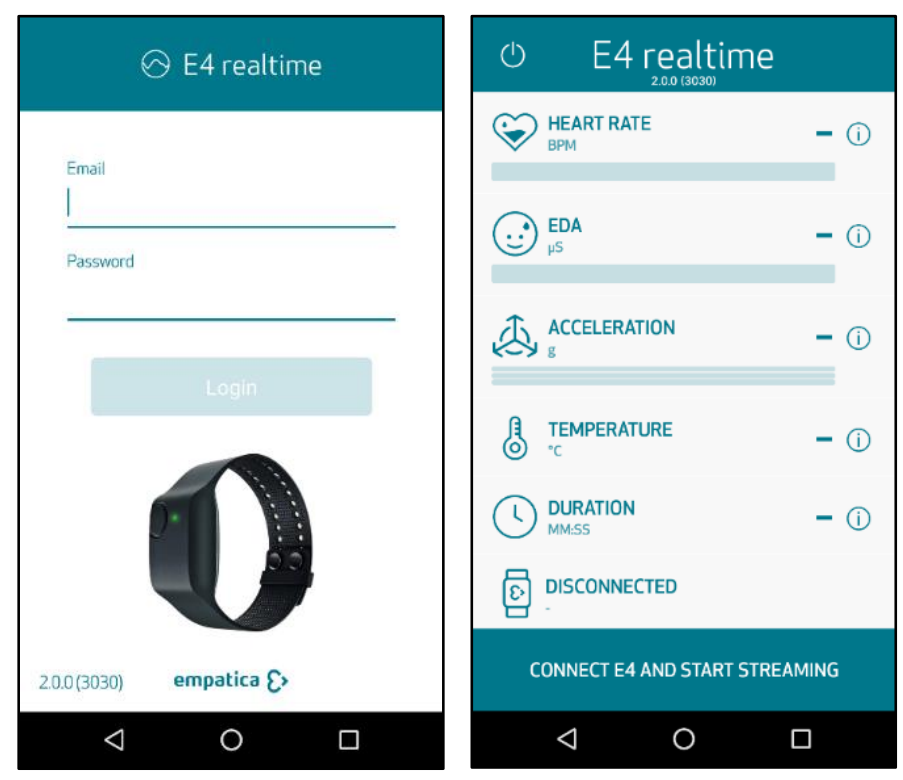

Figure 3.4: E4 Realtime smartphone application for Android phone

Empatica provides a web application interface Empatica Connect where

researchers can create their user account, visualize the data, and download raw comma separated file format (.CSV) files for further analysis. Each session is saved as a separate entry in the online database.

\subsection{Lab Setup}

Our aim for this research is to collect meaningful accelerometer sensor data from E4 to predict risky behavior that the veterans might demonstrate. However, working with the high-risk veteran population suffering from PTSD raises serious and complex legal and ethical issues. At all times, the research team had to be watchful not to overstep the personal boundaries of the veterans. We had to be sensible to not make any rash statements and be respectful towards the veterans. Although we had IRB approval to passively collect sensor data from high risk veterans, we decided it was not a rational nor 
an ethical decision to ask the high-risk veterans suffering from PTSD to wear the E4 device and wait for them to act dangerously in the real world in order to collect data for the research without some assurance that the system would work as we intended. Through this and other projects worked for over two years with high-risk veterans trying to understand them and their risky behavior. We did not want to push the veterans to any experience that would trigger their PTSD symptoms. It was not feasible to engage actual veterans in real-world data capture situations outside of the laboratory. The priority was to ensure that the veterans were not a threat to themselves or others.

Because of these considerations, the practical approach we settled on was to simulate aggressive crisis events and anger outburst moments in a veteran's life using actors based on the initial detailed ethnographic view on veteran's lived experience with anger that we obtained. To collect physiological data, we needed to find out what gestures, signs, postures, movements and speeches the veterans were most likely to use in a real scenario of an anger outburst or a risky behavior. We wanted to illustrate a few scenarios where the actors would be able to replicate those actions and mimic the veterans to a degree of satisfactory confidence. We emphasized on collecting the most accurate accelerometer sensor data of gesture and movements along with other physiological data. 


\section{CHAPTER 4}

\section{DATA COLLECTION}

\subsection{E4 Data Collection}

Three actors in turn wore the wearable wrist device recreated the thirteen gestures. To secure the E4 device it is advised to connect one snap at a time. It is important to make sure the E4 wristband band is tight enough to ensure the EDA electrodes do not change position on the skin during normal movement but not so much as to constrict blood flow or cause any discomfort. We asked them to perform each specific gesture at least three times. The gestures included 1) making fist in the air, 2) showing the middle finger, 3) putting both hands on the head, 4) Italian street talk gestures, 5) making a knife hand, 6) pointing at co-artist, 7) poking the chest of a coartist, 8) pounding the fist on a table, 9) shove a co-artist, 10) sweeping things off the table, and 11) throwing things on the table for example, money, keys, water bottles etc. Foot gestures like tapping foot, kicking objects and shaking leg came up during our ethnographic study. However, we did not ask the actors to act these gestures out, since we only had one E4 device which the actors wore on their wrist. It would have been great if we could use multiple E4 devices while collecting data. The actors were expressing aggressive behavior, anger, frustration, irritation, and throughout the sessions. This also allowed us to collect physiological data like their cardiac cycle, blood volume pulse, heart rate variance and galvanic skin response along with the 3-axis accelerometer sensor data. As mentioned earlier the scenarios involved three states. It started off with a high intensity anger condition. Then continued to a graduated anger condition scenario and ending with a non-anger but intense emotion condition. The rationale for the three stages 
was to ensure the gestures were enacted in a way approximating anger in real life, three scenes from plays were selected, each involving dialogue between the actor wearing the e4 and another actor. Each scene was specifically selected to get at different types of human experiences with high intensity emotion. One was explosive anger, the second scene dealt with a buildup of anger, and the third involved expressive, intense emotion that was not driven from anger. With time we could clearly see the PPG sensor, GSR sensor picking up data in higher frequency, which indicated the actors were demonstrating a physiological state which simulates that of a real-life scenario where one shows aggressive behavior.

Researchers including me and another graduate student from the computer science team were collaborating the data collection, while anthropologist, clinical physiologist and veterans were also present in the room. These data collection sessions were video recorded. A stopwatch was set and recorded as an additional time point reference to map the time in the video clips to the gesture. We connected the E4 device to the smartphone application E4 Realtime to observe the instantaneous data collection and streaming. An instance of data streaming is shown in figure 4.1 . 

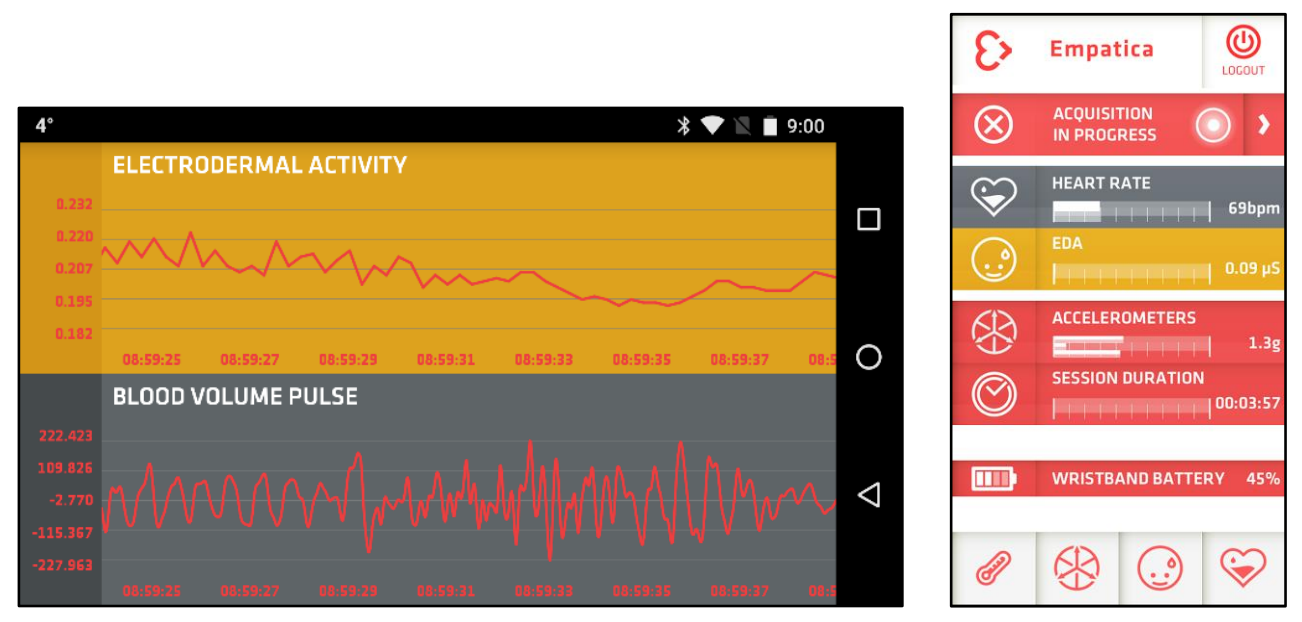

Figure 4.1: Mobile App user interface while live streaming the data to the server

We tapped the event marker button on the E4 as soon as the data collection

finished and stopped video recording. The timestamp from the event marker button later helped us to label the data with respect to the gestures. Data was storage and synchronization with the online storage E4 connect happened right away in the background since the phone was connected to the Wi-Fi.

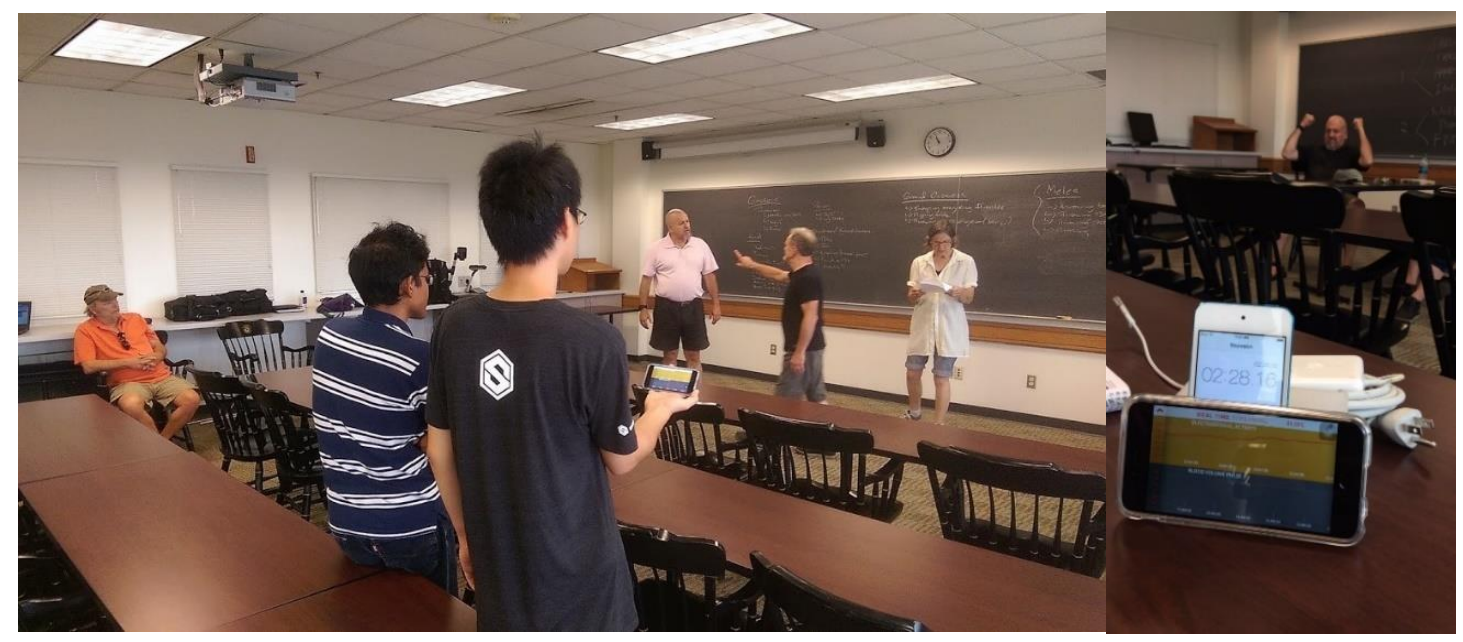

Figure 4.2: Data collection and Mobile App user interface for streaming the data to the server 
Figure 4.2 shows a moment captured while we collected data. Cold war veteran Mark Flower (on the far left) is seen observing the flow of events and doing a member check. Researchers visualizing the instantaneous data from E4 on the phone application. On the right, a moment when actor Bill poses for the hands-on-head gesture. We loggedin to the E4 connect account to confirm the sessions were recorded and data collection was successful. The E4 produces 7 downloadable .csv file which are ACC.csv, BVP.csv, EDA.csv, HR.csv, IBI.csv, TEMP.csv, tags.csv. These files hold data from the 3-axis accelerometer sensor, blood volume pulse, electrodermal activity (also known as Galvanic Skin Response - GSR), heart rate variance, body temperature and event marker timestamps data respectively. The data is secured with encryption and can be deleted after use [24]. For more information please refer to Appendix A. We will focus our attention on the ACC.csv file for this thesis and try to find out data clusters from them to identify gestures.

\subsection{Data Pre-processing}

To preprocess the information we collected, a single database was created that included data from all the sensor. It was observed that the six data (.csv) files stored data in different frequencies with different units. As an initial approach for data preprocessing we tried to normalize the data by bringing every sensor data (i.e. the six .csv files) to one common time series frequency. For example, the accelerometer sensor captures 32 readings every second. Whereas the PPG sensor captures heart pulse count every second. We attempted to normalize each sensor data in per second $(\mathrm{Hz})$ distribution. Issues that arose initially with this approach included concerns such as the need to have time-synced video matched to the physiological data feed from the E4. Since we are only interested in 
the accelerometer data, we did not explore that route.

\subsubsection{Time Synchronization}

The E4 device time-stamps each data point at all times. The process of timestamping depends on the method of recording. When it is connected to the smartphone application E4 Realtime over Bluetooth, in streaming mode, the E4 derives the time from the phone. However, if it is not linked to the application (in memory recording mode), when the E4 is connected to a computer via USB cable the time-stamp is labeled according to the computer's system clock. To avoid the drift of the clock during powering the device on and off, we waited for a while before capturing data.

\subsubsection{Timestamps in Empatica Data Files}

This file stores time in Unix time format which is different than that of a standard Excel file. The Unix time format starts time count from 01 JAN 1970 at 00:00:00 Coordinated Universal Time (UTC). On the other hand, an Excel file stores time from 01 JAN 1900. Date and time is stored as an integer value and decimal value respectively [29]. We needed to convert the Unix time to a format which would suit the MS Excel environment. The formula to convert the date time in Excel is-

$$
=(\mathrm{A} 1 / 86400)+\text { DATE }(1970,1,1)
$$

However, the E4 device does not consider the time zone offset while it stores data on Empatica Connect. It synchronizes all the data with the smartphone clock or the 
computer system clock. To include the time zone offset and synchronize data time-stamp, we use the following formula in Excel-

$$
=(\mathrm{A} 1 / 86400)+\mathrm{DATE}(1970,1,1)+(-5 / 24)
$$

Here the -5 indicates that time zone is synchronized to the US Eastern Daylight Time (EST) [30]. The offset can be changed to CST, PST etc. according to the requirement of the project.

\subsubsection{Mapping Gesture from Video Clips}

Now that we have the raw accelerometer data from E4 in .csv file format which are time-stamped to the system clock of the devices it is connected to (smartphone or computer), our next step is to label these data points with the thirteen gestures that the actors performed. After three sessions of data collection, we had more than 127 thousand data points from 5 sensor readings. The E4 device starts capturing accelerometer data points along $\mathrm{X}, \mathrm{Y}$ and $\mathrm{Z}$ axis as soon as the device is turned on. As a result, there were data collected in the beginning and end of each session which were not meaningful for this research. The entire data collection process was video recorded. The E4 device has its built-in timeline, the second timeline we had for our reference were the video clips. Looking back to the video clips we were able to determine start and end time of each 13 gestures of. Start and end time of 38 gestures enacted by the three actors (13 by actor one, 12 by actor two, 13 by actor three) were noted down in an Microsoft Excel file named "Video marked time action.xlsx". 


\begin{tabular}{|c|c|c|c|c|c|c|c|c|c|}
\hline 4 & A & B & c & D $\quad E$ & $F$ & G & H & 1 & \\
\hline 1 & Subject 1 & 11:17:00+Timer-29.05s & & Subject 2 & $2: 00+$ Timer-169.53s & & Subject 3 & 10:42:00+Timer-154.18s & \\
\hline 2 & & Start & End & & Start & End & & Start & End \\
\hline 3 & ThrowingMoneyOnBar & 20 & 22 & Throwingkeys & 40.75 & 41.57 & ThrowingMoneyOnBar & 2.02 & 3.65 \\
\hline 4 & & 28.71 & 30 & & 46.72 & 47.67 & & 12.71 & 14.4 \\
\hline 5 & Throwingkeys & 40.02 & 41.29 & & 52.34 & 53.57 & & 21.3 & 22.9 \\
\hline 6 & & 46.4 & 47.72 & HandsOnHead & 58.85 & 65.26 & Throwingkeys & 63.45 & 64.67 \\
\hline 7 & & 53.36 & 55.43 & & 67.23 & 75.73 & & 72.4 & 73.64 \\
\hline 8 & HandsOnHead & 60.67 & 75.17 & & 77.76 & 91.82 & & 80.43 & 82.37 \\
\hline 9 & ItalianStreetTalk & 79.26 & 80.76 & ItalianStreetTalk & 92.7 & 95.32 & HandsOnHead & 95.84 & 98.35 \\
\hline 10 & & 82.7 & 84.26 & & 95.37 & 96.56 & & 103.15 & 106.18 \\
\hline 11 & & 87.09 & 89.31 & & 97.28 & 98.43 & & 111.75 & 114.54 \\
\hline 12 & & 94.31 & 96.31 & & 101.2 & 102.9 & ItalianStreetTalk & 122.51 & 124.79 \\
\hline 13 & KnifeHand & 96.56 & 98.65 & KnifeHand & 108.03 & 109.21 & & 129.18 & 131.97 \\
\hline 14 & & 99.48 & 100.26 & & 111.46 & 117.15 & & 136.63 & 140.12 \\
\hline 15 & & 100.31 & 100.73 & & 119.2 & 121.06 & KnifeHand & 153.97 & 157.9 \\
\hline 16 & & 100.81 & 101.42 & Pointing & 125.12 & 131.43 & & 161.63 & 165.3 \\
\hline 17 & & 102.84 & 103.7 & & 131.65 & 133.03 & & 168.65 & 172.47 \\
\hline 18 & & 104.62 & 107.29 & & 137.71 & 138.8 & MiddleFinger & 177.63 & 179.02 \\
\hline 19 & Pointing & 121.25 & 122.12 & & 139.53 & 140.06 & & 185.53 & 187.06 \\
\hline 20 & & 122.03 & 122.4 & & 140.24 & 142 & & 193.84 & 199.02 \\
\hline 21 & & 123.68 & 124.26 & FistlnAir & 143.56 & 144.93 & Pointing & 209.44 & 215.86 \\
\hline 22 & & 124.51 & 124.86 & & 145.75 & 147.06 & & 218.68 & 222.78 \\
\hline 23 & & 127.56 & 127.9 & & 150.93 & 152.36 & & 227.09 & 230.58 \\
\hline 24 & & 127.91 & 128.71 & GivingTheFingers & 154.94 & 155.86 & FistlnAir & 234.8 & 236.22 \\
\hline 25 & & 129.26 & 130.15 & & 158.75 & 161.52 & & 239.84 & 241.68 \\
\hline 26 & FistlnAir & 133.25 & 135.36 & & 164.81 & 167.62 & & 247.5 & 250.45 \\
\hline 27 & & 136.18 & 137.28 & PokingChest & 170.84 & 172.81 & PokingChest & 259.7 & 261.3 \\
\hline 28 & & 139.8 & 142.02 & & 175.56 & 180.37 & & 262.68 & 264.75 \\
\hline 29 & GivingTheFinger & 147.02 & 147.93 & & 181.3 & 184.87 & & 265.8 & 267.68 \\
\hline 30 & & 150.53 & 151.22 & Shove & 193.53 & 194.58 & & 267.91 & 269.33 \\
\hline 31 & & 151.43 & 152.13 & & 197.18 & 189.36 & & 271.5 & 274.31 \\
\hline 32 & & 154.12 & 155.3 & & 200.03 & 203.12 & Shove & 284.16 & 287.12 \\
\hline 33 & & 155.35 & 156.12 & PoundingFist & 207.21 & 208.62 & & 290.98 & 293.55 \\
\hline 34 & Pokignotherchest & 175.87 & 178.25 & & 211.37 & 212.63 & & 299.81 & 302.64 \\
\hline 35 & & 185.81 & 187.81 & & 218.52 & 220.56 & PoundingFist & 310.63 & 313 \\
\hline 36 & Shove & 197.75 & 199.28 & SweepingAllobejc & 223.47 & 224.75 & & 319.76 & 321.31 \\
\hline 37 & & 205.43 & 206.59 & & 238.62 & 239.84 & & 330.2 & 331.4 \\
\hline 38 & & 206.9 & 207.97 & & 247.84 & 248.72 & SweepingAllobejctsofTak & 340.6 & 341.95 \\
\hline 39 & & 208.21 & 209.91 & Throwingthings & 269.73 & 271.06 & & 358.15 & 359.5 \\
\hline
\end{tabular}

Figure 4.3: Mapping gestures from video clip

The first column has the name of the gesture, the next two columns displays the start and end time in figure 4.3. For example, if we look at column $\mathrm{E}$ in the figure above, we will see that the first gesture performed by actor 2 was throwing keys on the table. Actor 2 performed this gesture three times sequentially. Let us tag these gestures as TM_a, TM_b and TM_c. The gesture TM_a was recorded from the 40.75th second to the 41.57th second of the video clip. We noted that down for each of the 38 individual gestures.

\subsection{Data Description}

The Empatica E4 device collects several physiological and accelerometer data using its sensors. The figure 4.4 shows the general output of the all sensor data that is captured. It shows the EDA sensor collecting galvanic skin response at the top, below 
that the blood volume pulse is displayed. Next is the accelerometer data. There are 4 graph lines for the accelerometer data. The accelerometer data is followed by the heart rate and body temperature time series data graph. Finally, at the bottom, the time-stamp is displayed. The red vertical line indicates that the event marker button was pressed.

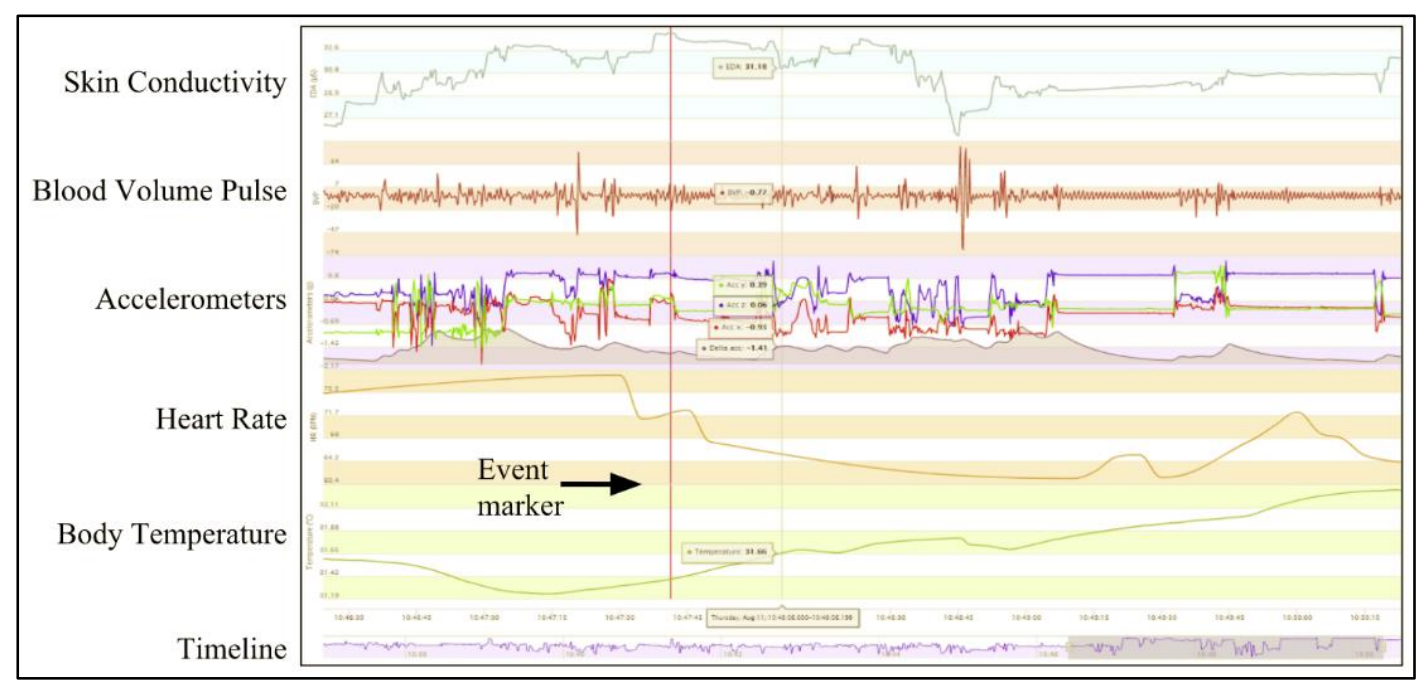

Figure 4.4: General output of the sensors data from Empatica E4

A short statistic of the data collected is presented in the table 4.1. A total of 127,858 data points were captured during the data collection sessions with the actors. The count of the data points from the sensors EAD, BVP, ACC, HR and TEMP of Subject 1, 2 and 3 are 52,076; 37,891; and 37,891 respectively. It is notable that research memberactor 1 collected approximately 14 thousand more data points compared to subject 2 and 3. This was because initially both the research team and the actors were trying to figure out how the data is being captured and how to act out the gestures. The process became much easier and smoother in time with actor 2 and actor 3. 


\begin{tabular}{|l|l|l|l|ll|}
\hline \multicolumn{2}{|c|}{ Actor 1} & \multicolumn{2}{c|}{ Actor 2 } & \multicolumn{2}{c|}{ Actor 3 } \\
\hline Sensor & Data count & Sensor & Data count & Sensor & Data count \\
\hline EAD & 1,982 & EAD & 1,442 & EAD & 1,442 \\
BVP & 31,748 & BVP & 23,102 & BVP & 23,102 \\
ACC & 15,872 & ACC & 11,552 & ACC & 11,552 \\
HR & 488 & HR & 353 & HR & 353 \\
TEMP & 1,986 & TEMP & 1,442 & TEMP & 1,442 \\
\hline Total Count & 52,076 & Total Count & 37,891 & Total Count & 37,891 \\
\hline Total Time & 8 min $15 \mathrm{sec}$ & Total Time & 6 mins & Total Time & 6 mins \\
\hline
\end{tabular}

Table 4.1: Summary of E4 sensor data collection

\subsubsection{ACC.csv file}

The figure 4.5 is an example of accelerometer data capture form E4 Connect. The dimensional axes (X, Y and $\mathrm{Z}$ ) data are represented with three different color, violet, red and green. Along with the three-axis accelerometer data E4 provides the moving average of acceleration force [31]. It is represented with purple graph.

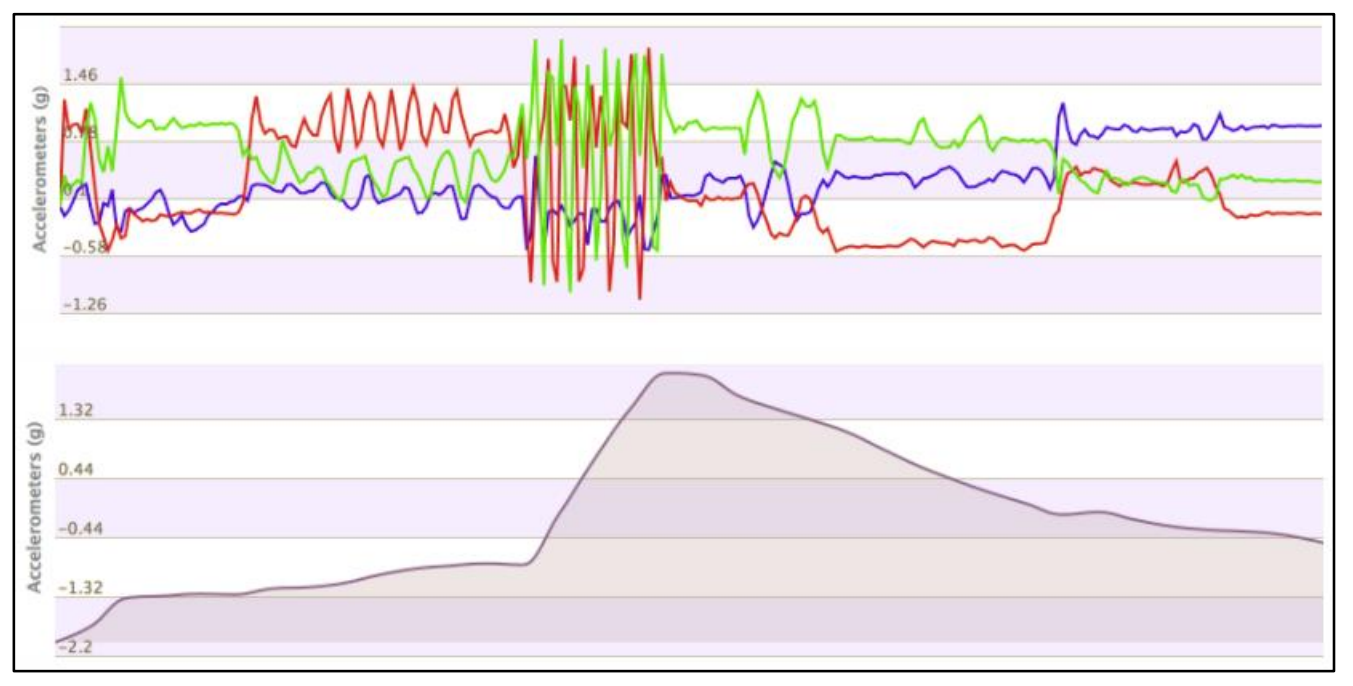

Figure 4.5: The dimensional axes and moving average of ACC 
There are three columns in the raw ACC.csv file downloaded from Empatica Connect. The first row of the file is an integer number that stores the date and time. This marks the beginning of the data collection (Figure 4.6). The following rows are data from $\mathrm{x}, \mathrm{y}$, and $\mathrm{z}$ axis are respectively in first, second, and third column. Data was collected for 6 to 8 minutes for each actor. All together there were 38,976 rows of accelerometer data points from all three actors combined. Each second 32 readings are stored in the data file. That is a collection of data over 20 minutes $(38976 / 32=1218$ seconds, 1218 seconds $=$ 20.3 minutes). The second row of the file indicates the frequency of data collection, which is 32 reading per second. The maximum value for the axes is 127 and the minimum value is -128 . For analytic purpose the values of the accelerometer reading in $1 / 64$ of the actual $g$ value. To convert these value to the actual g scale of -2 to +2 each cell needs to be multiplied by $1 / 64$. For example, $-128 *(1 / 64)=-2$.

\begin{tabular}{|c|c|c|c|c|}
\hline \multicolumn{2}{|c|}{ E5 } & $x$ & $\vee \quad f_{x}$ & \\
\hline 4 & $\mathrm{~A}$ & B & $\mathrm{C}$ & $\mathrm{D}$ \\
\hline 1 & 1472141771 & 1472141771 & 1472141771 & \\
\hline 2 & 32 & 32 & 32 & \\
\hline 3 & -60 & 0 & 25 & \\
\hline 4 & -60 & 0 & 24 & \\
\hline 5 & -60 & 0 & 24 & \\
\hline 6 & -61 & 0 & 23 & \\
\hline 7 & -60 & 0 & 23 & \\
\hline 8 & -60 & 0 & 23 & \\
\hline 9 & -60 & 0 & 23 & \\
\hline
\end{tabular}

Figure 4.6: Raw ACC.csv file from Empatica Connect 


\subsection{Data Labeling}

\subsubsection{Unix Time to Central Standard Time Conversion}

At this stage of the research the accelerometer data points were synchronized with the CST time zone. Details of the time synchronization is described in section 4.2.2. Figure 4.7 is a snapshot of how each data point was converted to from Unix time to CST time. Column $\mathrm{E}$ is the count of data point starting from 0 and continuing to 15869 . The first entry in column F is calculated by dividing value of cell E3 by the summation of cell $\mathrm{A} 2$ and $\mathrm{A} 1$. That is $\mathrm{F} 3=\mathrm{E} 3 / \$ \mathrm{~A} \$ 2+\$ \mathrm{~A} \$ 1$. The values are $0 / 32+1472139516$ which results to 1472139516.00 . This represents the time-stamp in Unix time. To convert this Unix time to CST we calculated the value in cell G3 with the formula $=(\mathrm{F} 3 / 86400)+$ DATE $(1970,1,1)+(-6 / 24)$. This gave us the result 09:38:36:00. That means the data collection for subject 1 started at 9:38 am and continued for 8 minutes till 9:46 am. Every 32 rows of the column $\mathrm{G}$ are color coded. This represents the 32 rows of data collected each second. We save the data in an Excel file named "ACC_subject_01.xlsx". The same steps were applied on the ACC.csv file of the two other subjects. 


\begin{tabular}{|c|c|c|c|c|c|c|c|}
\hline 4 & A & B & c & D & E & $\mathrm{F}$ & G \\
\hline 1 & 1472139516 & 1472139516 & 1472139516 & & Count & Unix Time & CST \\
\hline 2 & 32 & 32 & 32 & & & & \\
\hline 3 & -13 & -59 & 19 & & 0 & 1472139516.00 & $09: 38: 36.00$ \\
\hline 4 & -13 & -58 & 19 & & 1 & 1472139516.03 & 09:38:36.03 \\
\hline 5 & -13 & -58 & 19 & & 2 & 1472139516.06 & 09:38:36.06 \\
\hline 6 & -13 & -59 & 19 & & 3 & 1472139516.09 & 09:38:36.09 \\
\hline 7 & -13 & -60 & 20 & & 4 & 1472139516.13 & 09:38:36.13 \\
\hline 8 & -13 & -60 & 20 & & 5 & 1472139516.16 & $09: 38: 36.16$ \\
\hline 9 & -13 & -59 & 20 & & 6 & 1472139516.19 & 09:38:36.19 \\
\hline 10 & -13 & -58 & 20 & & 7 & 1472139516.22 & $09: 38: 36.22$ \\
\hline 11 & -13 & -59 & 20 & & 8 & 1472139516.25 & $09: 38: 36.25$ \\
\hline 12 & -13 & -62 & 21 & & 9 & 1472139516.28 & $09: 38: 36.28$ \\
\hline 13 & -13 & -61 & 22 & & 10 & 1472139516.31 & 09:38:36.31 \\
\hline 14 & -13 & -60 & 22 & & 11 & 1472139516.34 & 09:38:36.34 \\
\hline 15 & -12 & -59 & 22 & & 12 & 1472139516.38 & 09:38:36.38 \\
\hline 16 & -13 & -59 & 23 & & 13 & 1472139516.41 & 09:38:36.41 \\
\hline 17 & -13 & -58 & 23 & & 14 & 1472139516.44 & $09: 38: 36.44$ \\
\hline 18 & -14 & -58 & 21 & & 15 & 1472139516.47 & $09: 38: 36.47$ \\
\hline 19 & -14 & -59 & 21 & & 16 & 1472139516.50 & 09:38:36.50 \\
\hline 20 & -14 & -59 & 21 & & 17 & 1472139516.53 & $09: 38: 36.53$ \\
\hline 21 & -14 & -59 & 21 & & 18 & 1472139516.56 & $09: 38: 36.56$ \\
\hline 22 & -15 & -59 & 20 & & 19 & 1472139516.59 & 09:38:36.59 \\
\hline 23 & -14 & -60 & 21 & & 20 & 1472139516.63 & $09: 38: 36.63$ \\
\hline 24 & -13 & -58 & 20 & & 21 & 1472139516.66 & $09: 38: 36.66$ \\
\hline 25 & -13 & -58 & 19 & & 22 & 1472139516.69 & 09:38:36.69 \\
\hline 26 & -13 & -58 & 19 & & 23 & 1472139516.72 & $09: 38: 36.72$ \\
\hline 27 & -14 & -58 & 18 & & 24 & 1472139516.75 & $09: 38: 36.75$ \\
\hline 28 & -14 & -59 & 18 & & 25 & 1472139516.78 & $09: 38: 36.78$ \\
\hline 29 & -13 & -60 & 19 & & 26 & 1472139516.81 & 09:38:36.81 \\
\hline 30 & -12 & -60 & 20 & & 27 & 1472139516.84 & $09: 38: 36.84$ \\
\hline 31 & -12 & -60 & 21 & & 28 & 1472139516.88 & 09:38:36.88 \\
\hline 32 & -11 & -59 & 21 & & 29 & 1472139516.91 & $09: 38: 36.91$ \\
\hline 33 & -12 & -59 & 20 & & 30 & 1472139516.94 & $09: 38: 36.94$ \\
\hline 34 & -12 & -60 & 21 & & 31 & 1472139516.97 & 09:38:36.97 \\
\hline 35 & -11 & -58 & 22 & & 32 & 1472139517.00 & 09:38:37.00 \\
\hline 36 & -11 & -58 & 21 & & 33 & 1472139517.03 & $09: 38: 37.03$ \\
\hline 37 & -11 & -57 & 21 & & 34 & 1472139517.06 & $09: 38: 37.06$ \\
\hline 38 & -12 & -59 & 20 & & 35 & 1472139517.09 & 09:38:37.09 \\
\hline 39 & -13 & -61 & 21 & & 36 & 1472139517.13 & $09: 38: 37.12$ \\
\hline 40 & -14 & -59 & 21 & & 37 & 1472139517.16 & 09:38:37.16 \\
\hline 41 & -13 & -57 & 21 & & 38 & 1472139517.19 & 09:38:37.19 \\
\hline
\end{tabular}

Figure 4.7: Conversion of Unix Time to CST

\subsubsection{Mapping Gestures to Accelerometer Data}

Mapping the accelerometer data with the corresponding gestures was straight

forward. In section 4.2.3, we have shown how we mapped the start and end time of each gesture performed by the actors. Section 4.4.1 describes the process of mapping each accelerometer data point with a corresponding central time zone clock time. Taking the start and end time from Excel file video marked time action. $x l s x$ and the time-stamp from ACC_subject_01.xlsx we labeled the accelerometer data points with a matching gesture name as shown in figure 4.8 . 


\begin{tabular}{|c|c|c|c|c|c|c|}
\hline \multicolumn{2}{|c|}{ I10142 } & \multirow{2}{*}{ B } & $\checkmark \quad f_{x}$ & \multirow[b]{2}{*}{ D } & \multirow[b]{2}{*}{ E } & \multirow[b]{2}{*}{$\mathrm{F}$} \\
\hline & A & & C & & & \\
\hline 25 & $\mathrm{X}$-axis & Y-axis & Z-axis & Action & & \\
\hline 10133 & 2 & -68 & 16 & Pointing & & \\
\hline 10134 & 4 & -66 & $17 \mathrm{~F}$ & Pointing & & \\
\hline 10135 & -2 & -66 & 12 & Pointing & & \\
\hline 10136 & -7 & -63 & $15 \mathrm{~F}$ & Pointing & & \\
\hline 10137 & -1 & -59 & 20 & Pointing & & \\
\hline 10138 & -11 & -55 & 21 & Pointing & & \\
\hline 10139 & -22 & -54 & 12 & Pointing & & \\
\hline 10140 & -22 & -51 & $9 \mathrm{p}$ & Pointing & & \\
\hline 10141 & -21 & -48 & $6 \mathrm{~F}$ & Pointing & & \\
\hline 10142 & -25 & -49 & $6 \mathrm{~F}$ & Pointing & & \\
\hline 10143 & -22 & -49 & $9 \mathrm{~F}$ & Pointing & & \\
\hline 10144 & -17 & -49 & $12 \mathrm{~F}$ & FistInAir & & \\
\hline 10145 & -25 & -54 & $11 \mathrm{~F}$ & FistlnAir & & \\
\hline 10146 & -31 & -65 & $14 \mathrm{~F}$ & FistlnAir & & \\
\hline 10147 & -31 & -67 & $15 \mathrm{~F}$ & FistlnAir & & \\
\hline 10148 & -29 & -69 & $17 \mathrm{~F}$ & FistInAir & & \\
\hline 10149 & -36 & -81 & $17 \mathrm{~F}$ & FistInAir & & \\
\hline 10150 & -31 & -69 & $14 \mathrm{~F}$ & FistInAir & & \\
\hline 10151 & -25 & -63 & $11 \mathrm{~F}$ & FistInAir & & \\
\hline 10152 & -20 & -56 & $11 \mathrm{~F}$ & FistInAir & & \\
\hline 10153 & -17 & -58 & $14 \mathrm{~F}$ & FistInAir & & \\
\hline 10154 & -19 & -60 & $11 \mathrm{~F}$ & FistInAir & & \\
\hline 10155 & -19 & -66 & $8 \mathrm{~F}$ & FistlnAir & & \\
\hline 10156 & -16 & -68 & $7 \mathrm{~F}$ & FistlnAir & & \\
\hline 10157 & -16 & -63 & $5 \mathrm{~F}$ & FistlnAir & & \\
\hline 10158 & -18 & -55 & $6 \mathrm{~F}$ & FistInAir & & \\
\hline
\end{tabular}

Figure 4.8: Accelerometer data labeled with gesture

\subsection{Pinpoint Data Labeling}

Our aim was to feed the data we collected to a clustering algorithm to find out data clusters that identifies the gestures accurately. One of the principles of getting a good output from any machine learning algorithm is to provide the best quality of data available. The better a data set is, meaningful and superior results are. The data set we labeled with gestures up to this point were imprecise, since segments of the data are labeled with different gestures. For example, row 3 through row 1,152 is labeled as gesture- Fist in Air. However, this chunk of data rows does not accurately represent the three individual fists in air gestures that the actors performed. The E4 started capturing data as soon as the device was turned on. The device takes 10 to 15 seconds to calibrate the sensors, thus these are junk data. The actors did not start doing the gestures right after 
the calibration was done. There were times spent in between the performance. The gestures were not a continuous flow of events, while 32 rows of data were captured every second nonstop for 6 to 8 minutes. It was necessary to extract the core gesture data from more than 38 thousand data points. The need for removing the junk data pushed the research team to design a process of pinpoint data labeling.

\subsubsection{Step 1: Determine Start and End Time of Individual Gesture}

Let us take the data set of actor 1 to explain the process of pinpoint data labeling. We take the values from video marked time action.xlsx file and create a new file Pinpoint_Sub1.xlsx file. Figure 4.9 is a screenshot of the file where the gestures are label to the absolute preciseness. From Empatica connect we take the data capture start time offset (which is in this case 42 seconds) and note it down in cell O4. The difference between the start (cell M4) and end time (cell N4) is calculated and noted down in column P4. The next column Q4 is the actual start time of the corresponding gesture (Throwing Money on Bar, attempt 1). The time value is determined by adding the start time in cell M4 (video clip timestamp) with the offset previously set to 42 . Therefore, the precise pinpoint start time of the first gesture is noted in cell Q4 using the formula $=42+\mathrm{M} 4$, that is $42+2.02$ which equals to 44.02 . The next column R4 holds the end time of that gesture. It is simply calculated by adding the time difference from cell P4 with start time in $\mathrm{Q} 4$. That is, $=\mathrm{P} 4+\mathrm{Q} 4=1.63+44.02=45.65$. All the gestures are labeled with the start and end time in similar fashion. Whenever the time value exceeded 60, (i.e. 60 seconds $=1$ minute) we subtracted 60 from that value and noted the result in the next column. This process is repeated till the end of the file. 


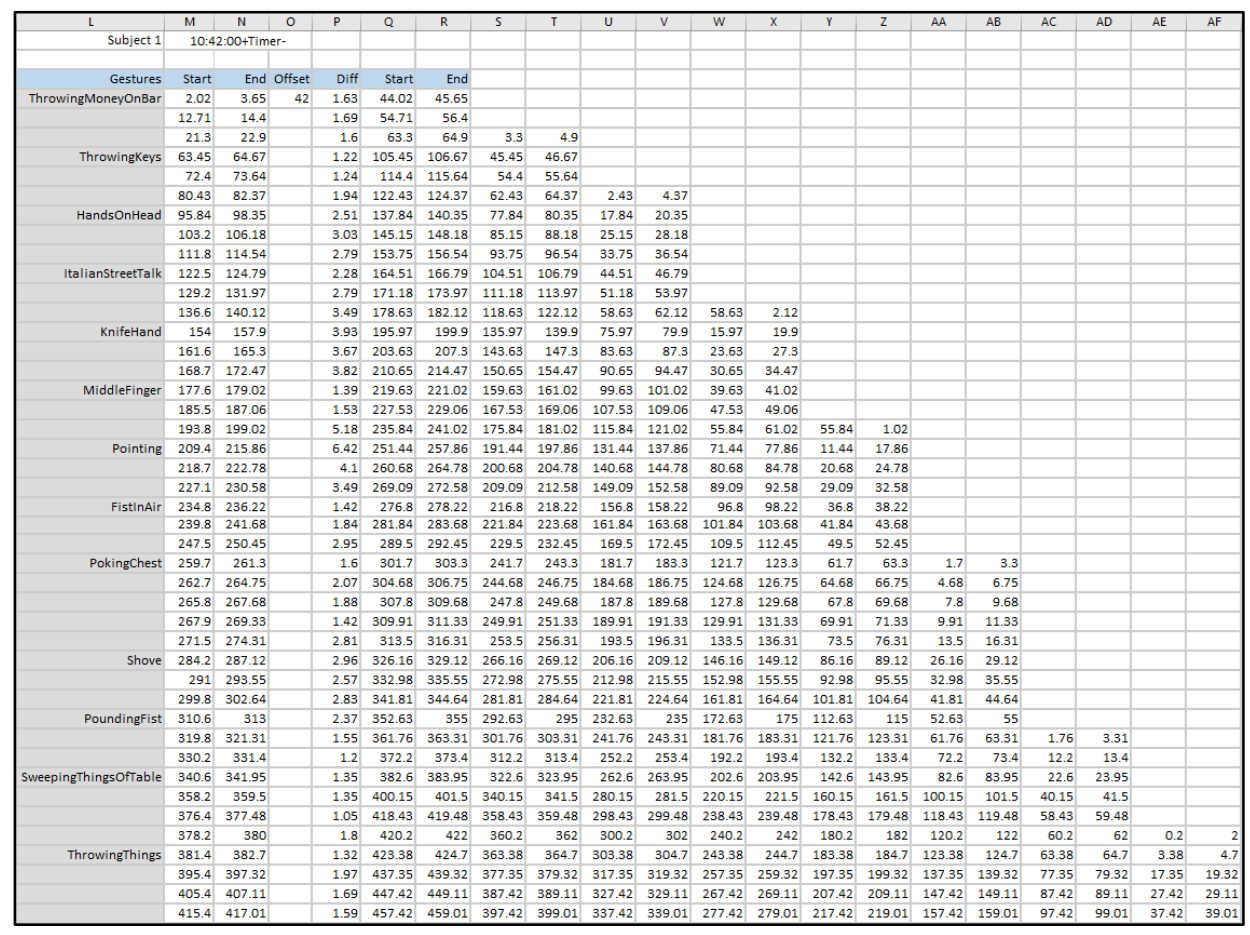

Figure 4.9: Step one of pinpoint data labeling (Subject 1)

\subsubsection{Step 2: Label Gestures in Central Standard Time Zone}

From Empatica connect and the ACC.csv file we know that the data collection for subject 1 began at 10:38:36.00 am. We determined an offset of 42 seconds, which brings the time to 10:39:18.00 am. These 42 seconds were taken by the E4 device to calibrate its sensors. The final start and end time noted earlier was 44.02 and 45.65 respectively. Therefore, based on our calculation the first gesture was recorded from the 44.02 second to 45.65 second of the $39^{\text {th }}$ minute of the $10^{\text {th }}$ hour. That is $10: 39: 44.02$ am to $10: 39: 45.65$ am in the morning. In this way the start and end time of each gesture was determined and saved in an Excel file. Figure 4.10 shows the second step of pinpoint data labeling for subject 3. We can easily determine the start time of data collection from E4 Connect. Data was being recorded from 11:16:36 am according to the central standard time zone 
(CST). The column in the far right is color coded with the clock time. The column on the left lists the gestures performed by the actors and the column next to it shows the number of times a gesture was performed.

\begin{tabular}{|c|c|c|c|c|c|c|c|c|c|}
\hline \multirow{2}{*}{4} & A & B & c & D & E & $\mathrm{F}$ & G & H & J \\
\hline & Subject 3 & & \multicolumn{6}{|c|}{ 11:17:00+Timer-29.05s } & \\
\hline 2 & & & \begin{tabular}{|c|} 
Video \\
Start
\end{tabular} & $\begin{array}{c}\text { Video } \\
\text { End }\end{array}$ & & $\begin{array}{c}\text { Time } \\
\text { Diff }\end{array}$ & $\begin{array}{l}\text { E4 Start } \\
\text { Time }\end{array}$ & $\begin{array}{l}\text { E4 End } \\
\text { Time }\end{array}$ & CS Time \\
\hline $3 T T$ & ThrowingMoneyOnBar & gesture 1 & 20 & 22 & 11:16 & 2 & 36 & 38 & $11: 16$ \\
\hline 4 & & gesture_2 & 28.71 & 30 & & 1.29 & 44.71 & 46 & \\
\hline 5 T & Throwingkeys & gesture_1 & 40.02 & 41.29 & & 1.27 & 56.02 & 57.29 & \\
\hline 6 & & gesture_2 & 46.4 & 47.72 & & 1.32 & 2.4 & 3.72 & $11: 17$ \\
\hline 7 & & gesture_3 & 53.36 & 55.43 & & 2.07 & 9.36 & 11.43 & \\
\hline 8 & HandsOnHead & gesture_1 & 60.67 & 75.17 & & 14.5 & 16.67 & 31.17 & \\
\hline 91 & ItalianStreetTalk & gesture_1 & 79.26 & 80.76 & & 1.5 & 35.26 & 36.76 & \\
\hline 10 & & gesture_2 & 82.7 & 84.26 & & 1.56 & 38.7 & 40.26 & \\
\hline 11 & & gesture_3 3 & 87.09 & 89.31 & & 2.22 & 43.09 & 45.31 & \\
\hline 12 & & gesture_4 & 94.31 & 96.31 & & 2 & 50.31 & 52.31 & \\
\hline $13 k$ & KnifeHand & gesture_1 & 96.56 & 98.65 & & 2.09 & 52.56 & 54.65 & \\
\hline 14 & & gesture_2 & 99.48 & 100.3 & & 0.78 & 55.48 & 56.26 & $11: 18$ \\
\hline 15 & & gesture_3 & 100.3 & 100.7 & & 0.42 & 56.31 & 56.73 & \\
\hline 16 & & gesture_4 & 100.8 & 101.4 & & 0.61 & 56.81 & 57.42 & \\
\hline 17 & & gesture_5 & 102.8 & 103.7 & & 0.86 & 58.84 & 59.7 & \\
\hline 18 & & gesture_6 & 104.6 & 107.3 & & 2.67 & 0.62 & 3.29 & \\
\hline $19 \mathrm{p}$ & Pointing & gesture_1 & 121.3 & 122.1 & & 0.87 & 17.25 & 18.12 & \\
\hline 20 & & gesture_2 & 122 & 122.4 & & 0.37 & 18.03 & 18.4 & \\
\hline 21 & & gesture_3 3 & 123.7 & 124.3 & & 0.58 & 19.68 & 20.26 & \\
\hline 22 & & gesture_4 & 124.5 & 124.9 & & 0.35 & 20.51 & 20.86 & $11: 19$ \\
\hline 23 & & gesture_5 & 127.6 & 127.9 & & 0.34 & 23.56 & 23.9 & \\
\hline 24 & & gesture_6 & 127.9 & 128.7 & & 0.8 & 23.91 & 24.71 & \\
\hline 25 & & gesture_7 & 129.3 & 130.2 & & 0.89 & 25.26 & 26.15 & \\
\hline $26 \mathrm{~F}$ & FistlnAir & gesture_1 & 133.3 & 135.4 & & 2.11 & 29.25 & 31.36 & \\
\hline 27 & & gesture_2 & 136.2 & 137.3 & & 1.1 & 32.18 & 33.28 & \\
\hline 28 & & gesture_3 3 & 139.8 & 142 & & 2.22 & 35.8 & 38.02 & $11: 20$ \\
\hline 29 & GivingThefinger & gesture_1 & 147 & 147.9 & & 0.91 & 43.02 & 43.93 & \\
\hline 30 & & gesture_2 & 150.5 & 151.2 & & 0.69 & 46.53 & 47.22 & \\
\hline 31 & & gesture_3 3 & 151.4 & 152.1 & & 0.7 & 47.43 & 48.13 & \\
\hline 32 & & gesture_4 & 154.1 & 155.3 & & 1.18 & 50.12 & 51.3 & \\
\hline 33 & & gesture_5 & 155.4 & 156.1 & & 0.77 & 51.35 & 52.12 & \\
\hline $34 \mathrm{P}$ & PokignOtherChest & gesture_1 & 175.9 & 178.3 & & 2.38 & 11.87 & 14.25 & \\
\hline 35 & & gesture_2 & 185.8 & 187.8 & & 2 & 21.81 & 23.81 & $11: 21$ \\
\hline $36 \mathrm{~s}$ & Shove & gesture_1 & 197.8 & 199.3 & & 1.53 & 33.75 & 35.28 & \\
\hline 37 & & gesture 2 & 205.4 & 206.6 & & 1.16 & 41.43 & 42.59 & \\
\hline 38 & & gesture 3 & 206.9 & 208 & & 1.07 & 42.9 & 43.97 & \\
\hline
\end{tabular}

Figure 4.10: Step two of pinpoint data labeling (Subject 3)

\subsubsection{Step 3: Label Accelerometer Data with Gestures}

For the final step of pinpoint data labeling we will continue with the example of subject 3. From the previous steps we determined the precise start and end time of each gesture and mapped the those start and end time in CST zone. In figure 4.11 the start and end time for Throwing money gesture A starts from 11:16:36.00 and ends at 11:16:38.00. This particular gesture was recorded for only 2 seconds. Figure 4.12 demonstrates how 
the accelerometer data were labeled manually with the gestures. Additional labeling was

done in column $\mathrm{E}$ and $\mathrm{F}$ with the count of gesture and the subject identifier respectively.

\begin{tabular}{|c|c|c|c|c|c|c|c|}
\hline 798 & -23 & -70 & 25 & 797 & 1472141795.91 & 11:16:35.91 & \\
\hline 799 & -24 & -63 & 24 & 798 & 1472141795.94 & $11: 16: 35.94$ & \\
\hline 800 & -22 & -58 & 22 & 799 & 1472141795.97 & $11: 16: 35.97$ & \\
\hline 801 [ & -21 & -61 & 20 & 800 & 1472141796.00 & $11: 16: 36.00$ & Throw money A \\
\hline 802 & -21 & -60 & 21 & 801 & 1472141796.03 & $11: 16: 36.03$ & Throw money A \\
\hline 803 & -21 & -60 & 19 & 802 & 1472141796.06 & $11: 16: 36.06$ & Throw money A \\
\hline 804 & -20 & -61 & 13 & 803 & 1472141796.09 & 11:16:36.09 & Throw money A \\
\hline 805 & -18 & -64 & 12 & 804 & 1472141796.13 & $11: 16: 36.12$ & Throw money A \\
\hline 806 & -18 & -67 & 11 & 805 & 1472141796.16 & $11: 16: 36.16$ & Throw money A \\
\hline 807 & -19 & -64 & 10 & 806 & 1472141796.19 & 11:16:36.19 & Throw money A \\
\hline 808 & -20 & -61 & 10 & 807 & 1472141796.22 & $11: 16: 36.22$ & Throw money A \\
\hline 809 & -23 & -55 & 12 & 808 & 1472141796.25 & $11: 16: 36.25$ & Throw money A \\
\hline 810 & -25 & -52 & 12 & 809 & 1472141796.28 & $11: 16: 36.28$ & Throw money A \\
\hline 811 & -28 & -50 & 12 & 810 & 1472141796.31 & $11: 16: 36.31$ & Throw money A \\
\hline 812 & -35 & -46 & 16 & 811 & 1472141796.34 & $11: 16: 36.34$ & Throw money A \\
\hline 813 & -35 & -43 & 17 & 812 & 1472141796.38 & $11: 16: 36.37$ & Throw money A \\
\hline 814 & -36 & -43 & 17 & 813 & 1472141796.41 & $11: 16: 36.41$ & Throw money A \\
\hline 815 & -42 & -42 & 20 & 814 & 1472141796.44 & $11: 16: 36.44$ & Throw money A \\
\hline 816 & -46 & -42 & 21 & 815 & 1472141796.47 & $11: 16: 36.47$ & Throw money A \\
\hline 817 & -48 & -45 & 21 & 816 & 1472141796.50 & $11: 16: 36.50$ & Throw money A \\
\hline 818 & -49 & -54 & 21 & 817 & 1472141796.53 & $11: 16: 36.53$ & Throw money A \\
\hline 819 & -51 & -54 & 21 & 818 & 1472141796.56 & $11: 16: 36.56$ & Throw money A \\
\hline 820 & -51 & -50 & 23 & 819 & 1472141796.59 & $11: 16: 36.59$ & Throw money A \\
\hline 821 & -52 & -61 & 21 & 820 & 1472141796.63 & $11: 16: 36.62$ & Throw money A \\
\hline 822 & -50 & -62 & 19 & 821 & 1472141796.66 & $11: 16: 36.66$ & Throw money A \\
\hline 823 & -41 & -54 & 15 & 822 & 1472141796.69 & $11: 16: 36.69$ & Throw money A \\
\hline 824 & -31 & -55 & 14 & 823 & 1472141796.72 & $11: 16: 36.72$ & Throw money A \\
\hline
\end{tabular}

Figure 4.11: Step three of pinpoint data labeling

\begin{tabular}{|c|c|c|c|c|c|c|c|c|}
\hline 4 & A & & B & & c & D & $\mathrm{E}$ & $\mathrm{F}$ \\
\hline $1 \times$ & X-Axis & & Y-Axis & & Z-Axis & Gesture & Count & Subject \\
\hline 2 & & -14 & & -57 & & 22 FistlnAir & & 1 Subject_A \\
\hline 3 & & -14 & & -56 & & 22 FistinAir & & 1 Subject_A \\
\hline 4 & & -14 & & -56 & & 22 FistlnAir & & 1 Subject_A \\
\hline 5 & & -14 & & -56 & & 22 FistlnAir & & 1 Subject_A \\
\hline 6 & & -14 & & -56 & & 22 FistlnAir & & 1 Subject_A \\
\hline 7 & & -15 & & -56 & & 22 FistInAir & & 1 Subject_A \\
\hline 8 & & -15 & & -57 & & 22 FistlnAir & & 1 Subject_A \\
\hline 9 & & -16 & & -58 & & 21 FistlnAir & & 1 Subject_A \\
\hline 10 & & -16 & & -58 & & 21 FistInAir & & 1 Subject_A \\
\hline \multirow[t]{2}{*}{11} & & -19 & & -60 & & 22 FistlnAir & & 1 Subject_A \\
\hline & & ? & & ? & & $?$ & & 2 \\
\hline 8767 & & -59 & & -58 & & 48 ThrowThings & & 9 Subject_C \\
\hline 8768 & & -52 & & -49 & & 48 ThrowThings & & 9 Subject_c \\
\hline 8769 & & -37 & & -52 & & 54 ThrowThings & & 9 Subject_c \\
\hline 8770 & & -3 & & -72 & & 62 ThrowThings & & 9 Subject_c \\
\hline 8771 & & 12 & & -100 & & 25 ThrowThings & & 9 Subject_c \\
\hline 8772 & & 49 & & -71 & & 17 ThrowThings & & 9 Subject_C \\
\hline 8773 & & -21 & & -47 & & 14 ThrowThings & & 10 Subject_C \\
\hline 8774 & & -21 & & -42 & & 10 ThrowThings & & 10 Subject_C \\
\hline 8775 & & -17 & & -46 & & 9 ThrowThings & & 10 Subject_c \\
\hline 8776 & & -17 & & -56 & & 10 ThrowThings & & 10 Subject_C \\
\hline 8777 & & -17 & & -63 & & 11 ThrowThings & & 10 Subject_c \\
\hline 8778 & & -18 & & -65 & & 11 ThrowThings & & 10 Subject_C \\
\hline 8779 & & -16 & & -66 & & 12 ThrowThings & & 10 Subject_C \\
\hline 8780 & & -16 & & -62 & & 10 ThrowThings & & 10 Subject_c \\
\hline 8781 & & -17 & & -62 & & 10 ThrowThings & & 10 Subject_c \\
\hline 8782 & & -18 & & -61 & & 9 ThrowThings & & 10 Subject_c \\
\hline 8783 & & -18 & & -60 & & 10 ThrowThings & & 10 Subject_C \\
\hline 8784 & & -17 & & -57 & & 12 ThrowThings & & 10 Subject_c \\
\hline 8785 & & -17 & & -55 & & 11 ThrowThings & & 10 Subject_C \\
\hline 8786 & & -23 & & -54 & & 10 ThrowThings & & 10 Subject_C \\
\hline
\end{tabular}

Figure 4.12: Pinpoint labeled data 
A key observation here is how the number of rows labeled with the gestures drastically decreased. When the initial labeling was done we had over 38 thousand data points form the accelerometer data. However, after completing the accurately labeling the gestures for all three participants, we have 8810 data instances. Over 30 thousand data rows were discarded. It is clear that the discarded data points were junk data which had nothing to do with the gestures. This significantly increases the quality of the data we prepared for the in-depth analysis.

\subsection{Data Visualization}

The purpose of data visualization is to convey the information in a clear, concise and simple manner. We used Weka and MATLAB for data analysis and visualization. Let us visualize the labeled accelerometer data that we pre-processed. The figure 4.13 below is a collection of stacked bar chart generated using Weka visualization tool kit. Starting from the top going clockwise the first three images show data columns of accelerometer data $\mathrm{X}, \mathrm{Y}$ and $\mathrm{Z}$ dimensional axes. The $\mathrm{X}$-axis of the bar chart is the range of accelerometer data from -128 to 127 , and Y-axis represents the number of count. The $\mathrm{X}$-Axis and Z-Axis data are distributed mostly towards the center but slightly skewed to the left and right respectively. The Y-Axis data is more evenly distributed compared to the other two axes. 


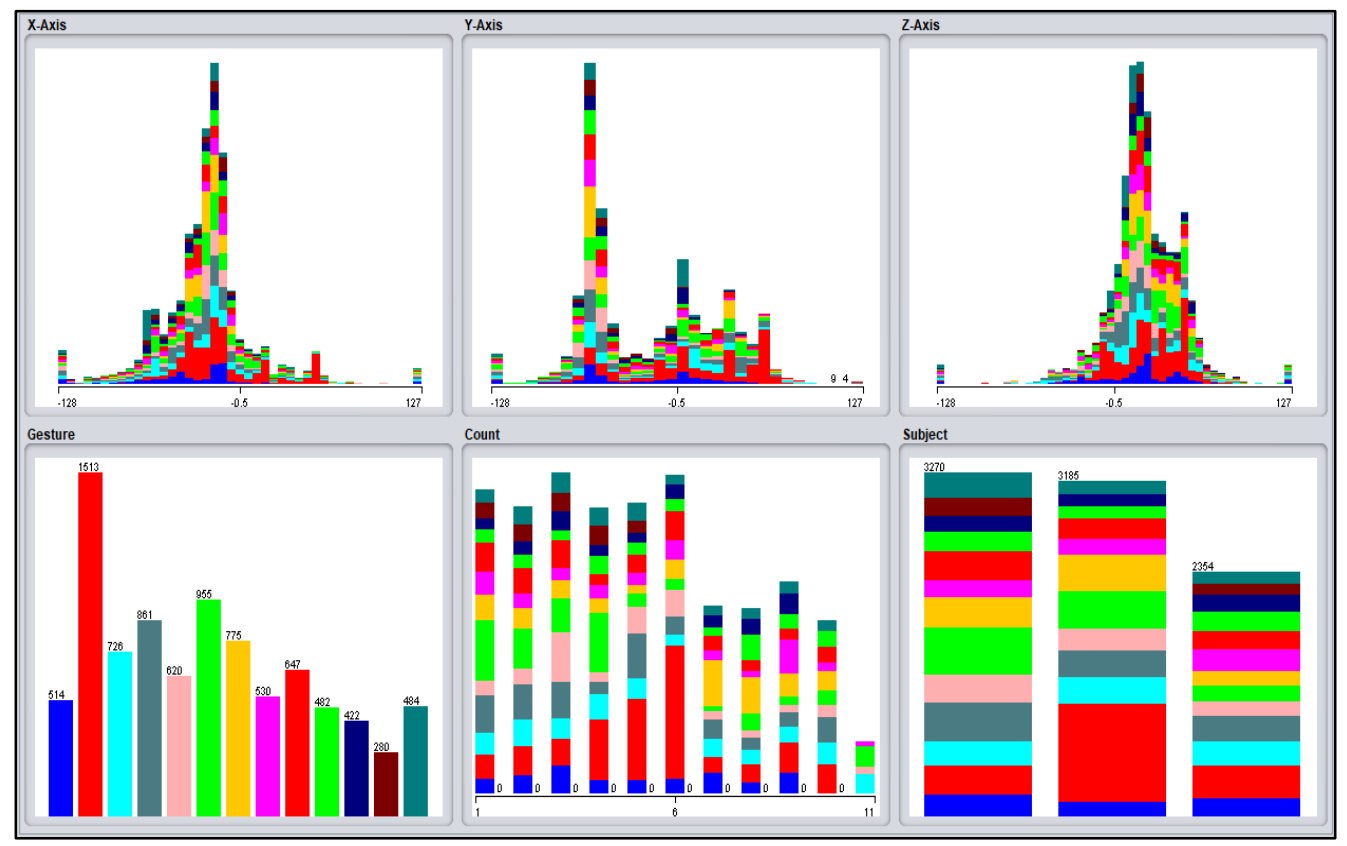

Figure 4.13: Visualization of the labeled accelerometer data form E4

The fourth bar chart displays the number of subjects along the $\mathrm{x}$-axis and count of gestures performed by each subject. There were three subjects sub_1, sub_2, sub_3 and they have $3270,3185,2354$ gesture counts respectively. The 13 different colors of the stacked bars signify the 13 gestures. It is to be noticed that sub_2 does not have the brown bar, which is throwing money on the bar. Subjects 1 and 3 performed 13 gestures each, and subject 2 did 12 of them. The fifth bar chart is the count of individual gestures. The colors represent the gestures. The sixth bar chart visualize the 13 gestures we labeled in the data set. The number on the top of each bar indicates the count of labeled row with respective gesture. The second bar in red has the highest count. This is the gesture Hands on Head. The least count is the tenth bar in brown, which is gesture of Throwing Money on the Table. 


\section{CHAPTER 5}

\section{DATA ANALYSIS}

Data analysis is the process by which some raw data is transformed or translated into a form of information that is structured, informative and easy to interpret. Data analysis allows its users to make decisions, answer questions, gain insight, test a hypothesis or disprove existing theory. [32, 33]. According to John, the process of collecting data, the technique of interpreting the results in such a manner that makes analysis easier along with high accuracy and preciseness in results is data analysis [34]. Statistical approaches play a huge role in data analysis. Our aim in this research is to identify clusters from accelerometer data from E4 device that are recognized as gestures veterans make while engaging in aggressive behavior. Once we obtain clusters we applied a supervised machine learning approach to classify the gestures and come up with a predictive model to predict aggressive risky behavior.

Using clustering model and expectation maximization (EM) algorithm we were able to cluster 12 unique gestures from the accelerometer data set we collected. Then we applied several classification algorithms among which k-nearest neighbors algorithm (k$\mathrm{NN}$ ) gave us the best result. By applying the k-NN algorithm we were able to classify the labeled dataset and came up with a predictive model to classify gestures we are tackling in this thesis.

\subsection{Expectation Maximization Algorithm}

The EM algorithm in statistics is an iterative method to find maximum likelihood (ML) when some of the data is missing. E-step and M-step are two processes for each iteration of the EM algorithm. In E-step, generally the observed data and current estimate 
of the model parameters is provided to estimate the missing data. This is done by explaining the choice of terminology using the conditional expectation.

In the M-step, it is assumed that the missing data are known. Data derived from the estimate computed in the E-step are used as the missing data to maximize likelihood function. In every iteration EM converges towards some local maximum causing the likelihood of the parameter to be increased in every iteration. [35, 36]

Number of uncertain parameters is an important factor to consider. Though it provides the best solution in ML sense, the best available guesses may not be acceptable as the number of uncertain parameters increases. As with each iteration, the algorithm converges towards a local maximum, the number of local peaks usually increases with multiple unknown (hidden/latent) parameters. Also, there are different procedures to find a global peak. One either should use different initial guesses for the estimated parameters or use more advanced method. The graph below shows the computation of the likelihood of each data point appearing under the current parameter guesses. We use probability density function. For example, data point at 1.761 is much more likely to be red $(0.189)$ than blue (0.00003). For each data point, we can turn these two likelihood values into weights: Red or Blue. 


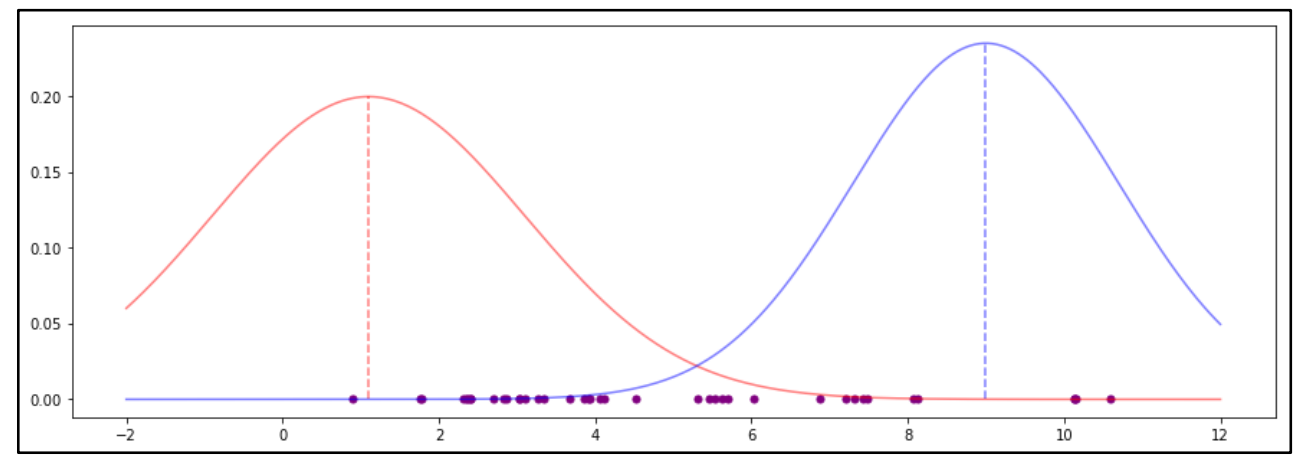

Figure 5.1: Principle of EM Algorithm

\section{2 k-Nearest Neighbor Algorithm}

$\mathrm{k}-\mathrm{NN}$ is a simple learning algorithm. It has been used since the 70's in statistical estimation and pattern recognition. $\mathrm{k}-\mathrm{NN}$ is a probabilistic function algorithm which uses a database to separate data points in classes to predict classification of new sample point. In k-NN, the data model structure is not determined from any assumptions on the underlying data distribution. Instead the structure is totally determined from the data. It's useful in the real world considering that data doesn't always follow the theoretical assumptions and it is considered as one of the first choices in scenarios where there is little or no previous knowledge of the distribution data. $\mathrm{k}-\mathrm{NN}$ is also known as a lazy algorithm because it doesn't do any generalization using the training data points. [37] No generalization means k-NN keeps all its training data and it needs all of the training data during the testing procedure. $\mathrm{k}-\mathrm{NN}$ also doesn't have explicit or a very minimal training phase. Though it's an algorithm known for its high accuracy, it's considered very resource hungry. As the algorithm stores all the training data, it's computationally expensive and the prediction stage can be slow (with big $\mathrm{N}$ ) with high 
memory requirement. Apart from these it's a very versatile algorithm, highly useful for classification or regression.

\subsection{Clustering Gesture Data}

We used Waikato Environment for Knowledge Analysis (Weka) version 3.8.1 machine learning suit to run the EM algorithm. The final data file we created for data analysis was named AllUserData_WithHeader.csv. This file stores the labeled data of all the gestures performed by three actors. There are 129 gestures in total of which 13 are unique. However, Weka does not allow us to import .csv files directly for analysis. The accelerometer data file was converted to .arff format which is WEKA friendly. Weka comes with a built-in csv to arff converter. To convert a .csv file to .arff format, users need to open the ArffViewer from the Tools tab on the Weka GUI Chooser. Click on File and open the .csv file on the arff viewer panel as shown in figure 5.2 (a). Save the file in .arff format. The accelerometer data file AllUserData_WithHeader.arff is now ready to be imported in Weka. Uses can select the Explorer option in the Weka GUI chooser. The explorer panel has options to run clustering and classification algorithms. It also provides the option for data processing, filtering and visualization. Figure 5.2 (b) is a screenshot of the Weka Explorer. The explorer panel summarizes the accelerometer data by lists the name and type of the attributes. The mean and standard deviation of the numeric attribute is shown in the Explorer panel. 


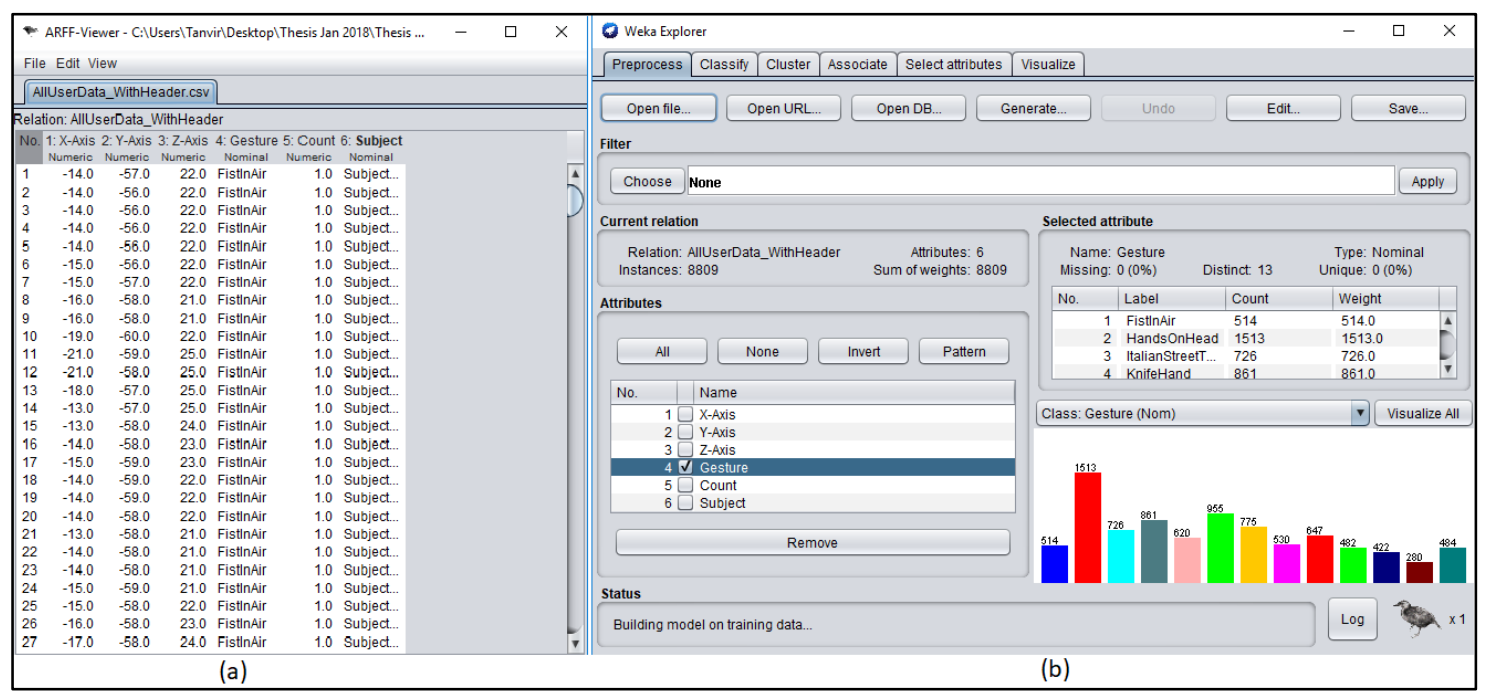

Figure 5.2: (a) Arff viewer in Weka (b) Weka Explorer

EM algorithm was applied on the accelerometer data set. A split of 75:25 ratio was chosen for training and testing the machine learning algorithm. The maximum number of iterations was 100 by default. When we visualize the output generated by the EM algorithm we have the option to plot the data points of accelerometer sensor's $\mathrm{X}, \mathrm{Y}$, $\mathrm{Z}$ axis along either of the $\mathrm{X}$ or $\mathrm{Y}$ axis of the output plane. One of the limitations of Weka is that it does not provide visualization in 3-dimensional space. Figure 5.3 is displaying the output from the EM algorithm. This clustering output is generated from the accelerometer data of all three subjects. The unsupervised machine learning algorithm was able to find out 12 clusters from the accelerometer data. Twelve different colors represent the 12 gestures. The figure displays a scatter plot of the $\mathrm{X}$-axis and $\mathrm{Y}$-axis of the accelerometer data points. Red, blue, green, cyan and pink clusters are observed marked in circles. These clusters are the strong clusters among all the others. The clusters are labeled with a likelihood value by the EM algorithm. These values determine 
if a cluster is a strong cluster or a weak one. Out of these twelve clusters representing gestures, the top five strong clusters we found were 1) knife hand, 2) pounding fist, 3) pointing, 4) throwing keys and 5) Italian street talk had the highest likelihood value and accuracy.

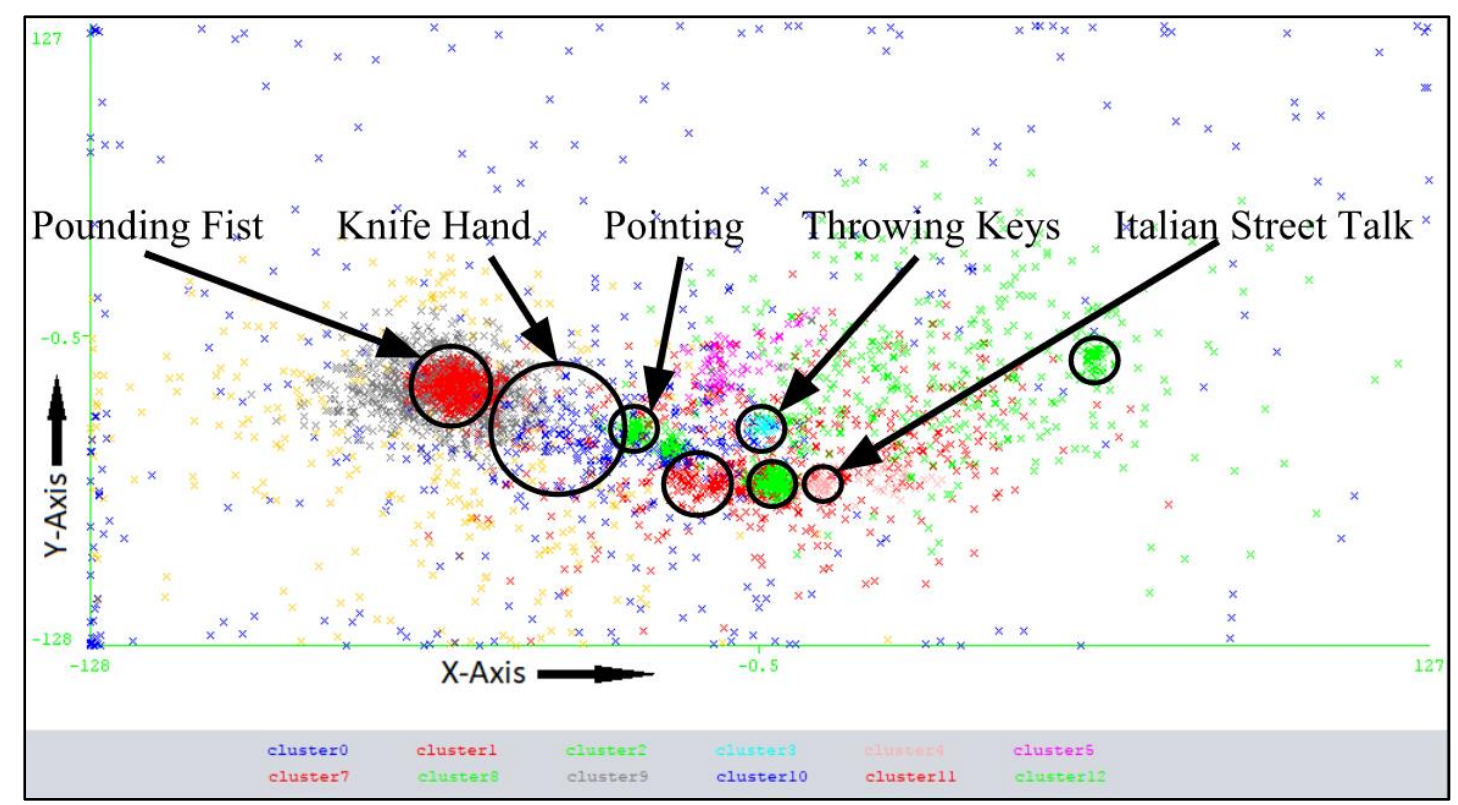

Figure 5.3: Output from EM algorithm showing clusters representing aggressive gestures

The accuracy of the identified clusters was $81.72 \%$. The table 5.1 lists the top five gesture clusters determined. Figure 5.4 is a screenshot of the output in Weka. 


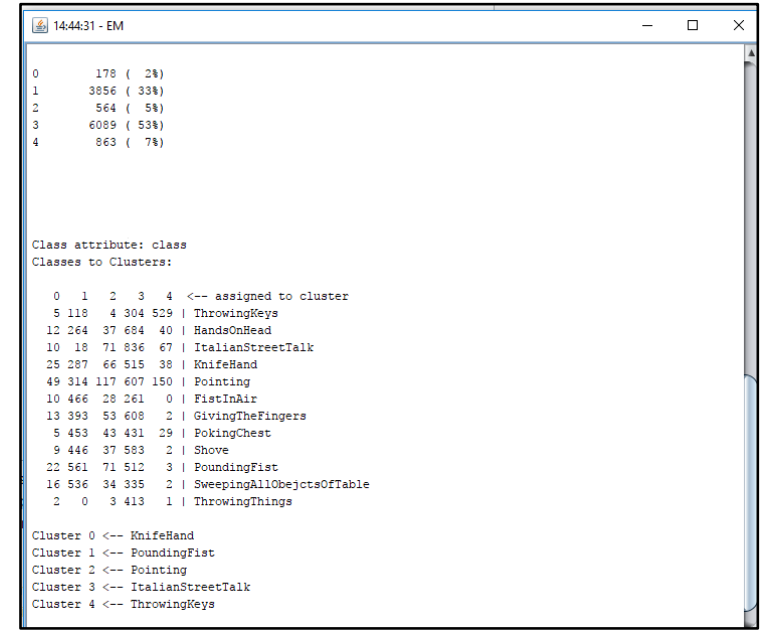

Figure 5.4: Output from EM algorithm in Weka

\begin{tabular}{|l|l|l|l|}
\hline Sl. No. & Clusters & Gesture clusters & Accuracy \\
\hline 1 & Cluster 0 & Knife Hand & $(0.93)$ \\
\hline 2 & Cluster 1 & Pounding Fist & $(0.87)$ \\
\hline 3 & Cluster 2 & Pointing & $(0.84)$ \\
\hline 4 & Cluster 3 & Throwing Keys & $(0.74)$ \\
\hline 5 & Cluster 4 & Italian Street Talk & $(0.69)$ \\
\hline
\end{tabular}

Table 5.1: Top five clusters identified representing the gestures

Figure 5.5 display the output from the data set of second actor participant. The accelerometer data was fed into the EM clustering algorithm separately. The algorithm was able to detect 9 clusters which represent gestures from the accelerometer data. Among the nine clusters the top three clusters were knife hand, pointing and pounding fist. These gestures are seen in the output in orange, blue and ash clusters. The output from all three participants together and the output from an individual participant throws 
the same clusters. Therefore, it can be concluded that the clustering algorithm detects meaningful gesture clusters on which we can rely on.

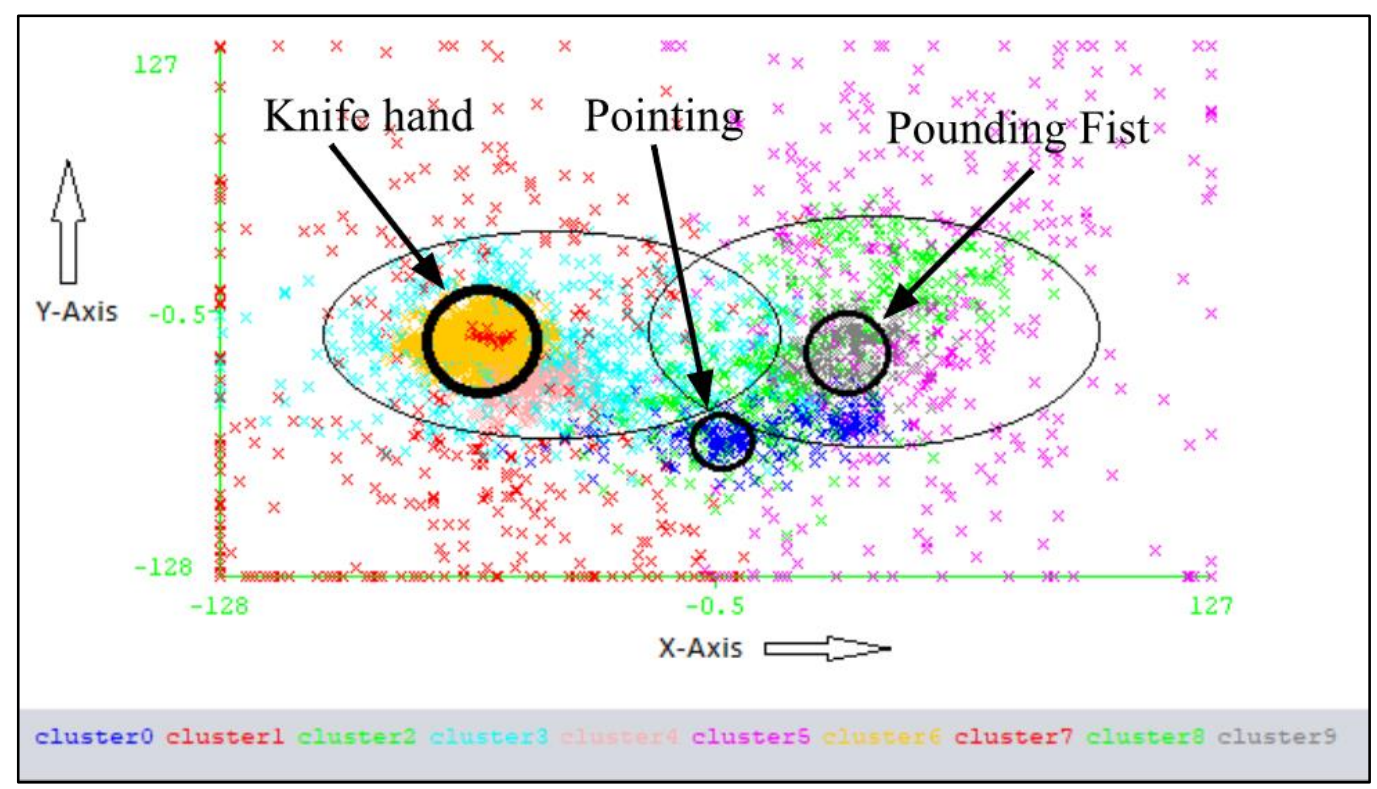

Figure 5.5: Gesture clusters output form EM algorithm. Participant 2

\subsection{Classification Algorithms - Supervised Learning}

The research team spent a significant amount of time to accurately label the accelerometer data. This allowed us to apply supervised learning technique and classify the data collected based to the labeled gestures. Several classification algorithms including variations of $\mathrm{k}-\mathrm{NN}$ algorithm and support vector machine (SVM) were applied on the pinpoint labeled data set. Our goal was to build a classification model that would be able to classify gesture from the accelerometer sensor and eventually modify the classification technique to a degree of preciseness so that it could be used as a predictive 
model to predict aggressive gestures. We used MATLAB (version R2017b) to run the classification algorithms and machine learning techniques. The MATLAB machine learning Toolkit was installed. Both .xlsx and .csv format files can be directly imported to MATLAB. Once we imported the AllUserData_WithHeader.csv fie open the classification learner app in MATLAB to run classification models. The data distribution along the $\mathrm{X}-\mathrm{Y}$ axis, $\mathrm{Y}-\mathrm{Z}$ axis and $\mathrm{X}-\mathrm{Z}$ axis are displayed in figure 5.7. The 13 gestures are labeled as classes and they are represented with different colors (Figure 5.6).

\begin{tabular}{|c|}
\hline Classes \\
\hline FistlnAir \\
\hline HandsOnHead \\
\hline ItalianStreetTalk \\
\hline KnifeHand \\
\hline MiddleFinger \\
\hline Pointing \\
\hline PokingChest \\
\hline PoundingFist \\
\hline Shove \\
\hline SweepOffTable \\
\hline ThrowKeys \\
\hline ThrowMoney \\
\hline ThrowThings \\
\hline
\end{tabular}

Figure 5.6: Classes labels

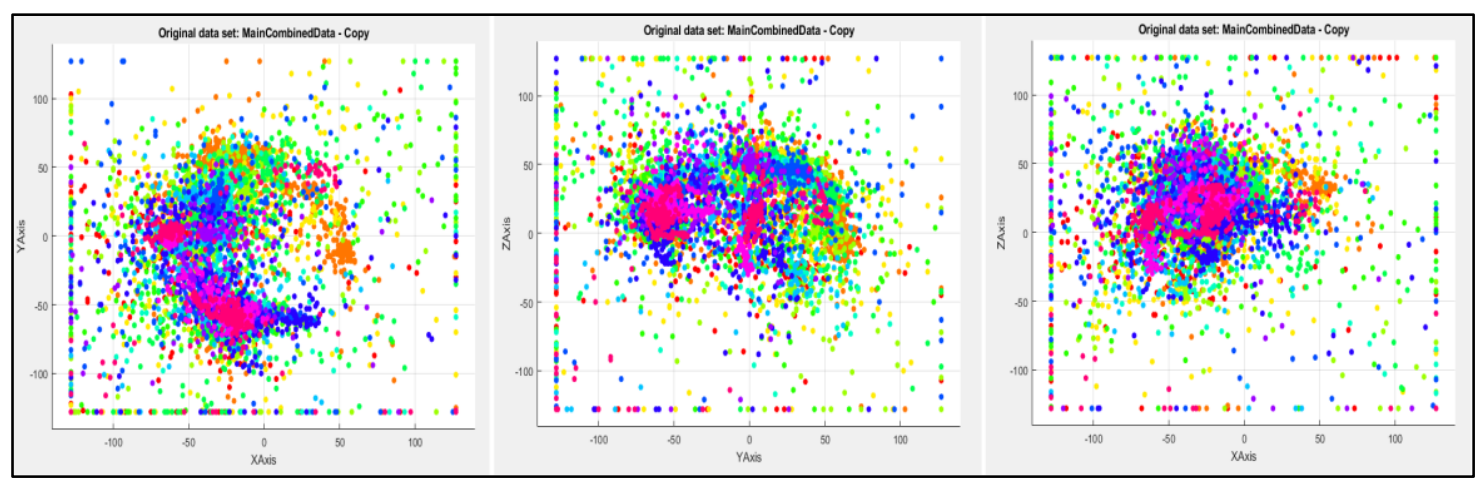

Figure 5.7: Scatter plot of accelerometer sensor data along different axes 


\subsubsection{Support Vector Machine}

SVM are supervised learning models used to analyze data for classification and regression analysis. A training data set is given where every data point is labeled with class labels. SVM is a non-probabilistic binary linear classifier. It is widely used in detection and recognition of hand gestures, facial gestures and active learning [38-41]. We applied the default quadratic SVM classifier on MATLAB and found the classification was accuracy rate was $23.6 \%$. This is a very low accuracy rate for classification. The accuracy of linear SVM was even worse, that is $19.9 \%$. Figure 5.8 shows the result of we obtained after running quadratic SVM on the accelerometer data set. The dots $(\bullet)$ represents correctly classified data point, and the cross $(\mathrm{x})$ represents the incorrectly classified data point on the scatter plot.

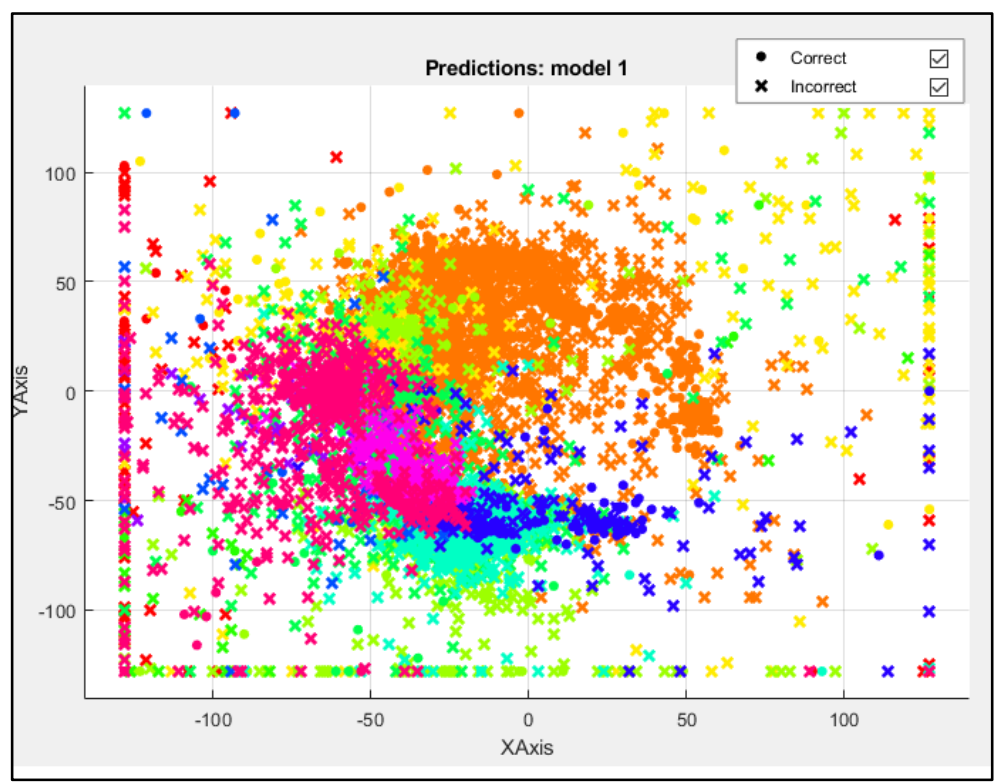

Figure 5.8: Classified data plot from SVM. Accuracy $23.6 \%$ 


\subsubsection{Distance Weighted k-nearest Neighbors}

$\mathrm{k}-\mathrm{NN}$ algorithm is another probability distribution method used to in classification and regression analysis. The output is a class member where the classes are known. A data point is classified based on a majority vote of its neighbor members or data points. The member is assigned to a class that is most common among the $\mathrm{k}$ nearest neighbor. Here $\mathrm{k}$ is an integer typically starting from 1. A simple example of $\mathrm{k}-\mathrm{NN}$ classification is shown in figure 5.9. The test sample in the center identified with the green circle is needed to be classified as either of the two classes, class 1 as blue square or class 2 as red triangle. When $\mathrm{k}=3$ the area taken under consideration is determined with the solid line circle. In this case the green circle is assigned to the second class, i.e. red triangle since there are 2 triangles and only 1 square inside the inner circle. In another condition when $\mathrm{k}$ $=5$, determined by the dashed line circle, the green circle member under classification algorithm is assigned to class 1 , i.e. blue square since there are three blue square inside the area covered by the dashed line circle. [37]

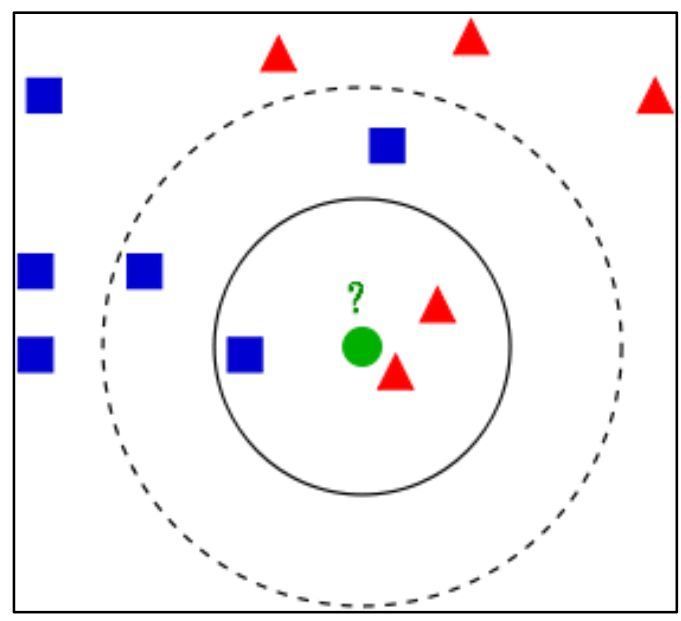

Figure 5.9: k-NN classification algorithm 
A variation of the k-NN algorithm is weighted k-NN. Here each member of the data set is assigned a weight based on the distance from the member that is needed to be classified. The weight can be assigned based on the distance, or on other characteristics. For example, when $\mathrm{k}=1$, the green circle in figure 5.9 is classified as either class 1 or class 2 . If the weight of the blue square is more than the summation of the weights of the two red triangles within the solid line circle, the green circle would be assigned to the blue square class. We applied weighted k-NN algorithm in MATLAB on our data set. The accuracy of correctly classifying the gesture data was $46.6 \%$. Which was more than what we found from SVM. Figure 5.10 (a) and (b) shows data points correctly and incorrect classified respectively in a scatter plot.

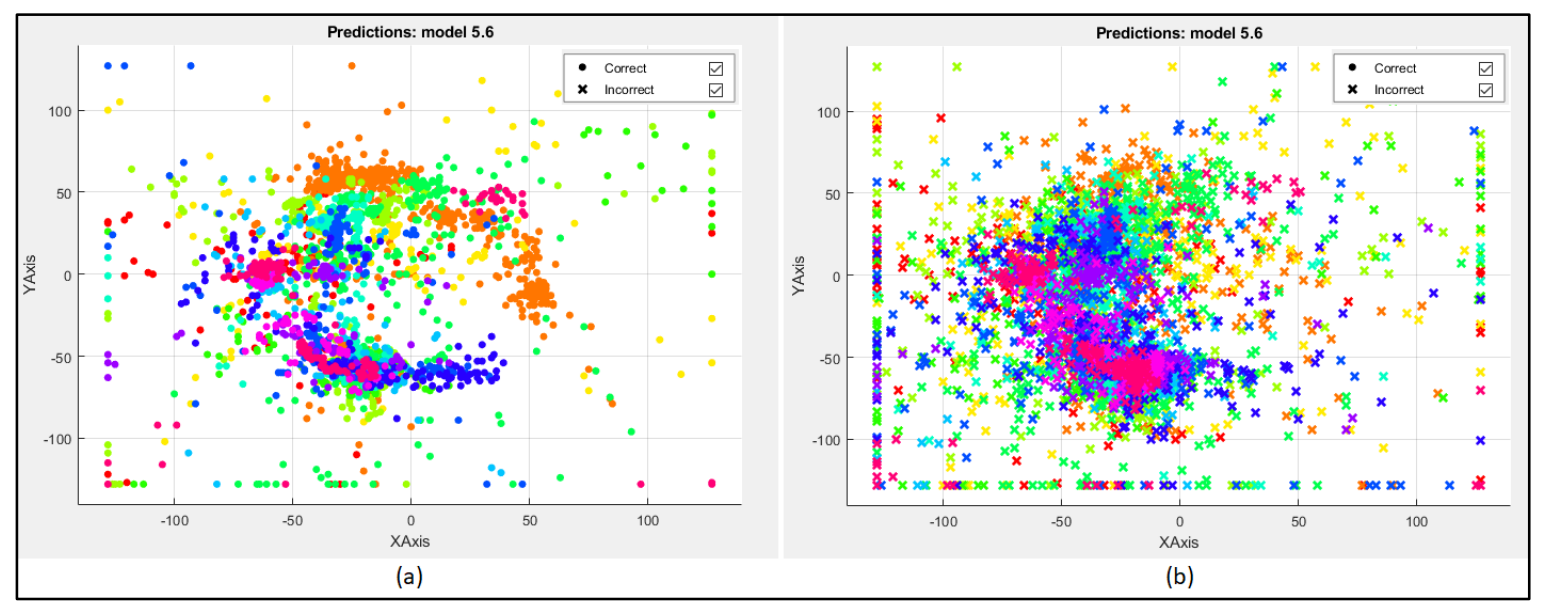

Figure 5.10: (a) Correctly classified data points. (b) Incorrectly classified data points

\subsection{3 k-nearest Neighbors $(\mathrm{kNN})$}

Lastly, the simplest machine learning algorithm the k-NN, was applied on the data set and this approach surprisingly obtained the highest accuracy in correctly classifying 
the accelerometer sensor data points. The accuracy of correctly classifying the data points to the gesture classes was $66.7 \%$. This indicates that out of hundred data points from the E4 accelerometer sensor data more than 66 data points were labeled correctly to the correct gestures. Figure 5.11 below shows the correctly classified gesture data points.

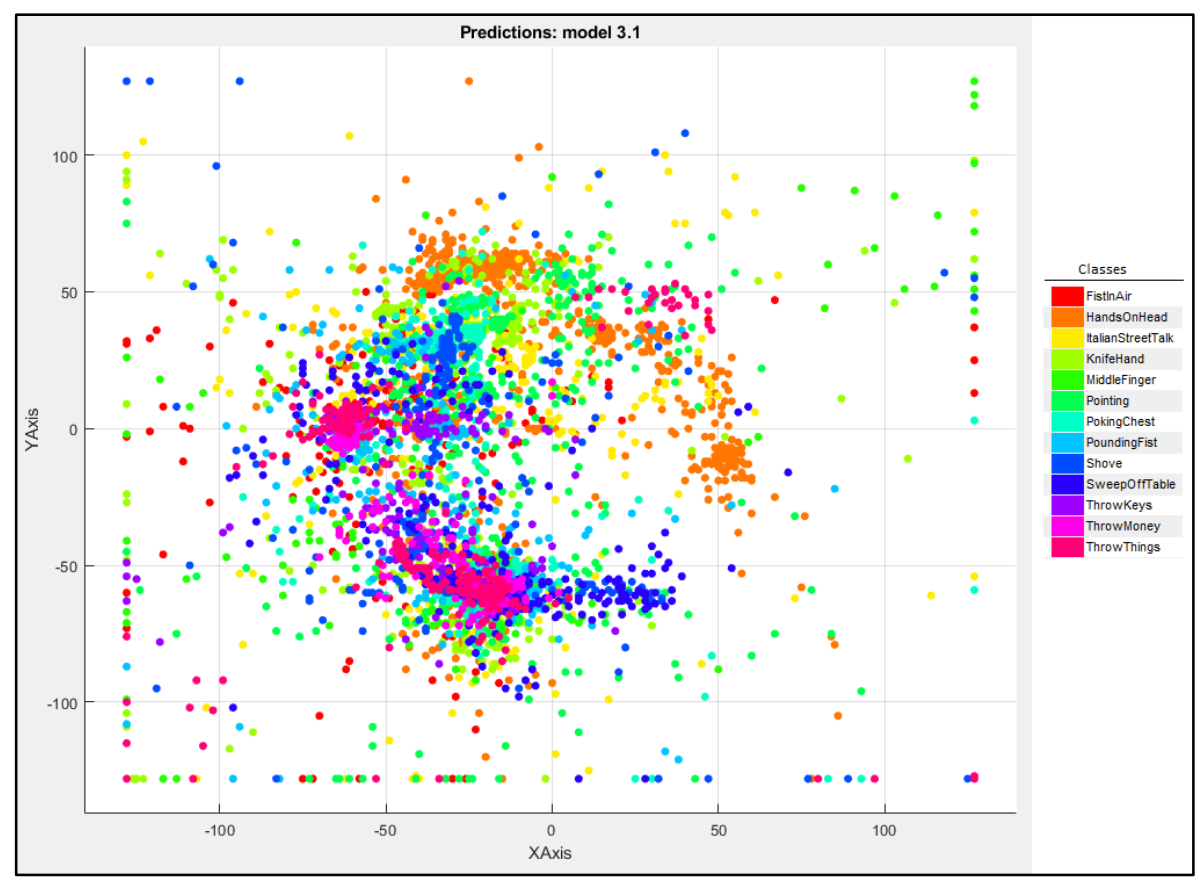

Figure 5.11: Correctly classified data points using k-NN algorithm. Accuracy 66.7\%

The image above is the scatter plot of all the correctly classified data points of the 13 gestures along $\mathrm{X}$ and $\mathrm{Y}$ axis. We can see most of the accelerometer data points are placed in the $2^{\text {nd }}$ where value of $\mathrm{X}$ is negative, and $\mathrm{Y}$ is positive; and $3^{\text {rd }}$ quadrant where the values of $\mathrm{X}$ and $\mathrm{Y}$ are both negative. To clear up the clutter we look at all the class labels (i.e. gestures) separately in figure 5.12. 


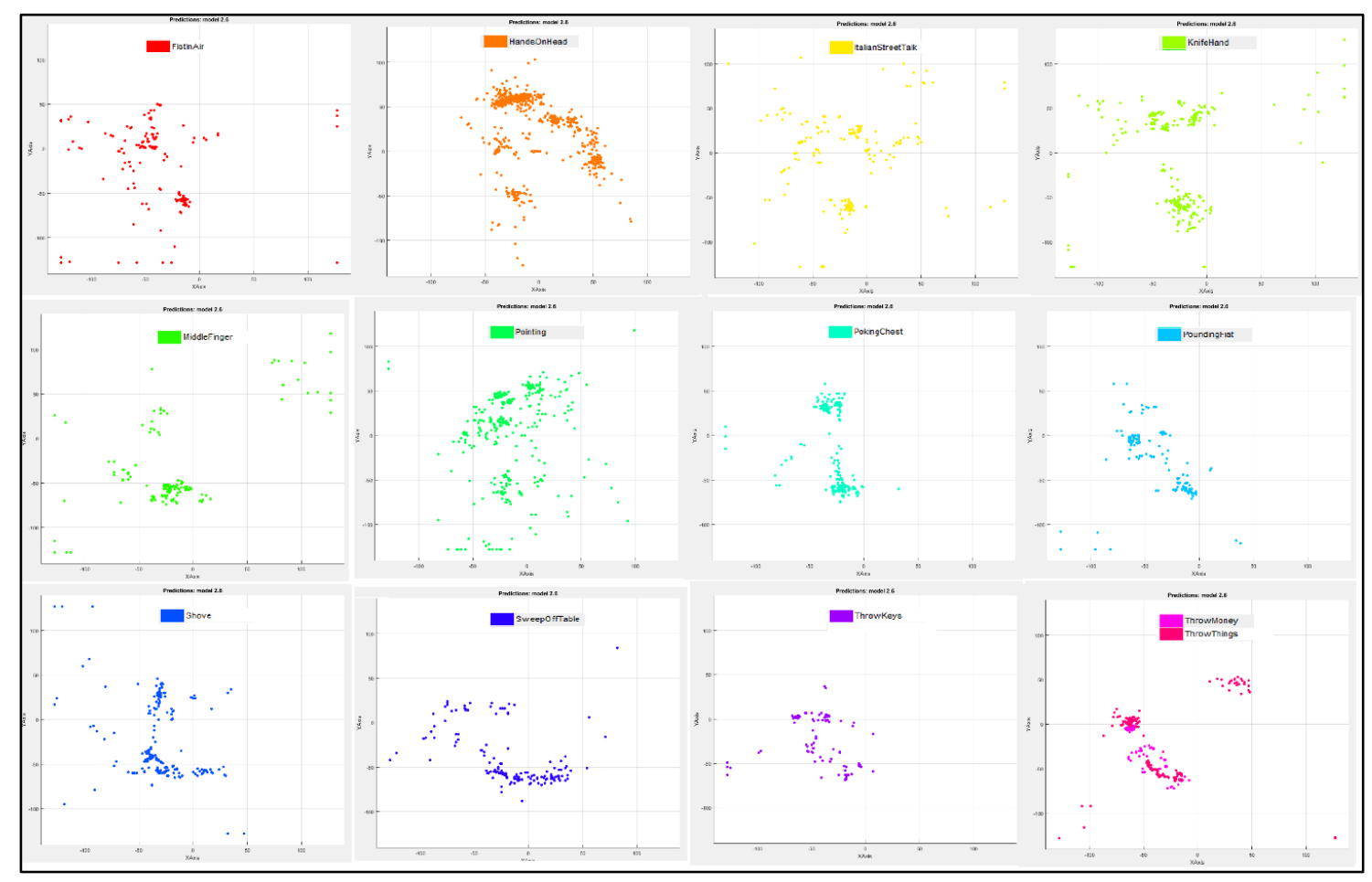

Figure 5.12: Classified data of 12 gestures plotted separately

This figure gives us a clear indication of the data points that are correctly classified. A point to be noted here is that there were 13 gestures in the class label when we trained the classifier model, however in figure 5.12 there are 12 graphs of the classified data. This is because we merged the last two gestures Throwing money and throwing things in the same graph shown in the very last box. Notice there are two shades of pink data instances labeled indicating the two separate gestures. Another insight we gained from here is that the two gestures of throwing money and things (book, water bottle etc.) are somewhat similar. It can be taken into consideration when recreating the data collection scenarios, that these two gestures are not very different from each other. The following two figures show the confusion matrix and the receiver operating characteristic (ROC) curve of the data set obtained after applying the k-NN algorithm. 


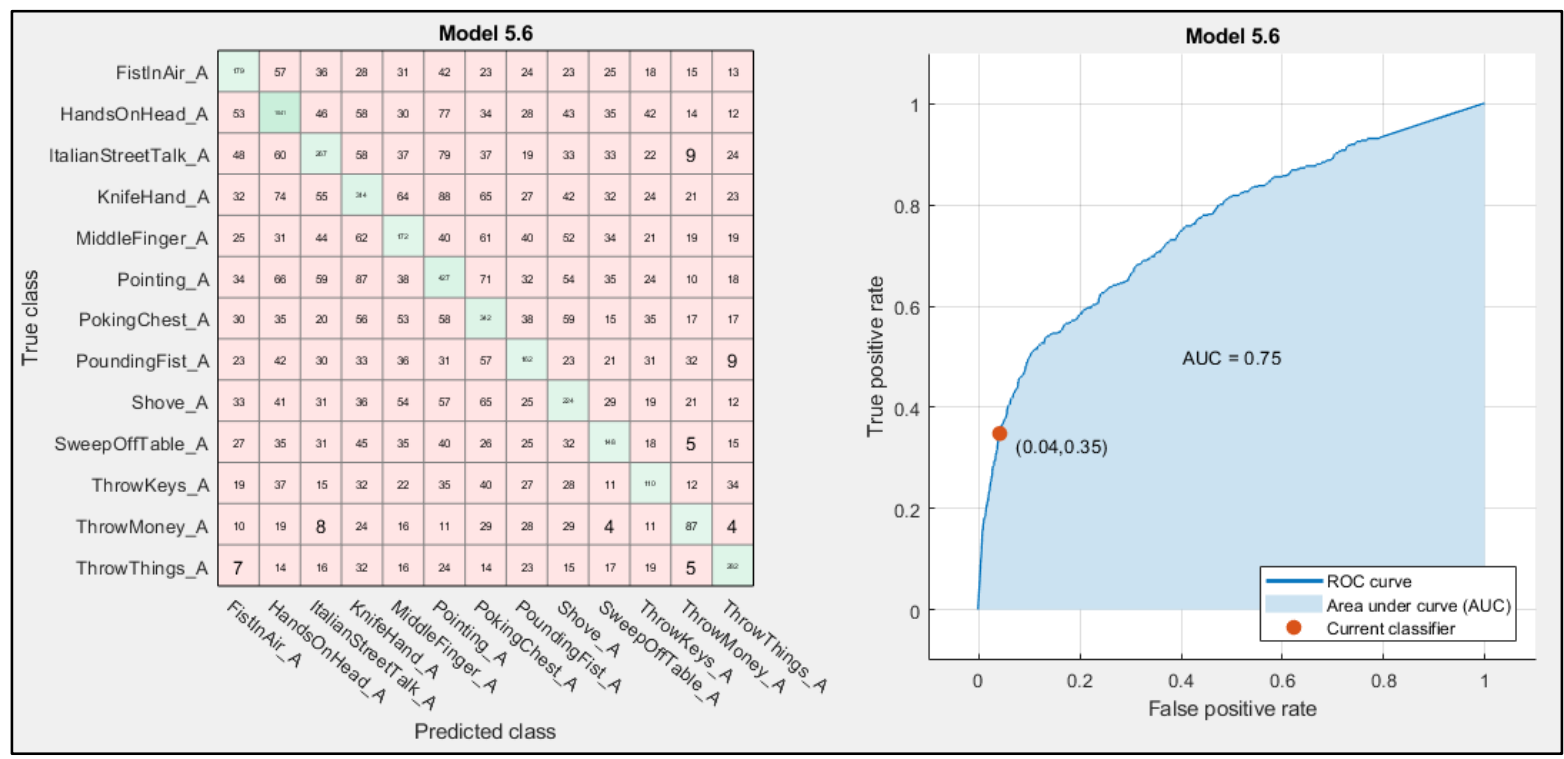

Figure 5.13: Confusion Matrix and ROC Curve 


\section{CHAPTER 6}

\section{RESULTS}

\subsection{Research Outcome}

We had four research aims (RA) when we started the research. Those were research aim 1) to be able to distinctively and reliably identify a set of clusters representing aggressive gestures. Research aim 2) was to find the strongest cluster and the smallest number of gesture from the thirteen gestures that are most informative. The third research aim 3) was to be able to label the accelerometer data to a level of accuracy where every gesture performed would be labeled down to the precision of seconds. The goal was to do a pin-point labeling of the accelerometer data and gestures. Finally, the fourth research aim was 4) to develop a predictive model with classifier algorithms that would predict a risky or aggressive behavior of a veteran by analyzing and classifying the gesture data from the E4 sensor.

\subsection{Distinctively and Reliably Identify a Set of Clusters Representing Aggressive Gestures}

The research team was able to find clusters from the data set we collected and related the clusters to gestures that the actors performed. Chapter 6 section 3 describes the machine learning technique for detecting clusters. We were able to detect 12 gestures after applying EM algorithm. Although there some of the clusters detected were very weak and had low likelihood value, the strong clusters in the output gave us confidence that the algorithmic approach is able to detect the gesture clusters. 


\subsection{Narrow Down to the Smallest Number of Gesture that are Most Informative}

After doing the data analysis and using EM clustering, we found 12 gesture clusters. However, they were not all very strong clusters. In order to identify clusters accurately with a degree of accuracy, we needed to find the strong clusters outputs. We focused on the top five gesture clusters from the result we got. These dense and strong clusters could instantly tell us which gesture cluster a given data point belongs to. The five clusters represent the following gestures (and accuracies), 1) knife hand $(0.93), 2)$ pounding fist $(0.87), 3)$ pointing $(0.84), 4)$ throwing keys $(0.74)$ and 5) Italian street talk (0.69). They are considered as strong clusters since they have a high likelihood value (value noted in parenthesis). This project presents the analysis of the gesture patterns of an individual that represents aggressive gestures. We found out that $81.72 \%$ of times our clustering model correctly identified the five gestures from the accelerometer data.

\subsection{Pin-Point Labeling of the Accelerometer Data and Gestures}

A great deal of research was done to label the accelerometer data from the E4 sensor with the gestures performed by the actors. Chapter 5 section 5 describes elaborately how the pin-point data labeling was done. Based on the calculations we did, we were able to accurately label 129 gestures with the start and end time of their occurrence. Thousands of rows of junk data were removed from the data set. The precise data labeling allowed us to get a better classification. The quality data set the foundation of a next research goal which it to build a predictive model that detect risky behavior from the aggressive gesture data form the E4 sensor. 


\subsection{Development of a Predictive Model with Classification Algorithms}

Applying the k-NN algorithm we were able to classify each gesture correctly with an accuracy of $67.7 \% .13$ gestures were indicated as the class variable. We used these class members (gestures) to train the classify the data set. This predictive model generated from MATLAB is now ready to run prediction on accelerometer data collected using the E4 device. It can be concluded that the k-NN is the best classification approach one can take to classify the gesture data. We tried out the weighted k-NN, linear SVM, quadratic SVM, Fine Tree, Ensemble boosted trees, classification techniques. However, those classification approaches did not give us satisfactory results. 


\section{CHAPTER 7}

\section{APPLICATION}

\subsection{Integration with QRF Smartphone Application}

The potential application of our research findings is quite broad. We have been able to identify aggressive gestures that the veteran suffering from PTSD make from the E4 sensor data. When the aggressive gestures are detected, an alert can be sent to the health care facility or law enforcement agencies warning about a probable risky behavior. This gesture detection process can be incorporated in developing a system that would integrate with the existing QFR smartphone application. The E4 device can be set up to be always connected to a veteran's smartphone. If the data analysis results from the E4 data can be incorporated with the QRF app to generate an alert triggering system, the QRF application can be designed to contact the peer-mentor of an at-risk veteran as soon as it predicts a risky behavior from the veteran's gestures. This alert could also notify family members when there is a probability of a veteran in engaging in a risky behavior where they themselves or others might be in danger.

\subsection{Elderly Care and Veteran Monitoring}

Another application of the research outcome can be to address a specific need for the senior citizens. Senior citizens or patients in a health care facility who face various challenges in their day to day life. In a retirement home, old age home or a nursing home a robot can be programmed to communicate the alert triggering system. In situations when no one is available to attend the person in need, a robot can easily intervene and trigger an alert to someone in charge and seek immediate attention. One of the projects in our lab is working on programming a Bioloid Premium Robot (figure 7.1) which 
responds to external sound sources from the environment. If an elderly person or a patient is wearing the $\mathrm{E} 4$ and the $\mathrm{E} 4$ data is sent to the robot, the robot could be programmed to run the predictive model of detecting risky behavior or crisis events. If the robot detects any crisis event, it would be able to send an alert and seek attention from the surrounding environment. A wearable device (E4) could connects to the robot wirelessly, enabling the robot to notify in real time, thus effectively increase response time to emergency situations. It is both a friendly companion and a reliable solution. The Bioloid Premium Robot can be assembled to make a humanoid form.

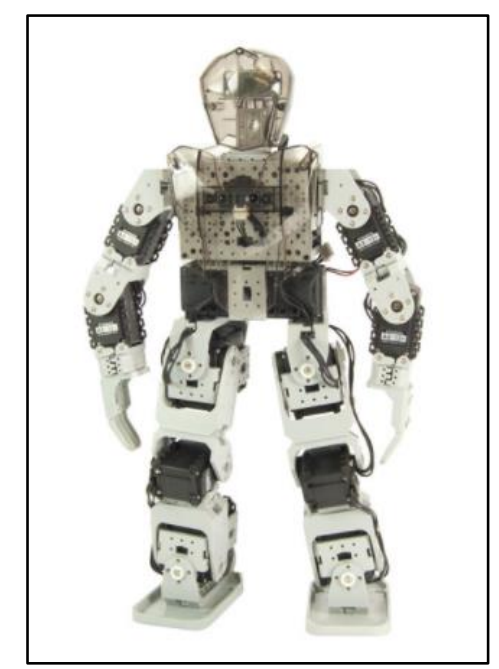

Figure 7.1: Bioloid Premium Robot

The research group working on the application of the robot were able to program the robot to move autonomously towards a sensor while avoiding objects in its path. The next step is to connect a wearable device to the robot to detect when an elderly person is experiencing any crisis event. The theoretical application of this thesis coupled with the robot project could be to move autonomously and connect to the E4 device. While the 
autonomous movement enables the robot to provide guidance to elderly person, it would also encourage the elderly person or veteran to be more trusting of the technology and more welcoming of this form of intervention. The helper and companion aspects of the robot address both the physical and emotional health of the senior and veteran community. 


\section{CHAPTER 8}

\section{CONCLUSION}

\subsection{Contribution of This Thesis}

This research contributed in the science of studying human behavior, focusing on the life of US veterans. Veterans suffering from PTSD or other war trauma engage more in risky behaviors and crisis events compared to the civilian population. By conducting an ethnographic research, the thesis was able to develop a general taxonomy of warning signs (scenarios, social settings, and gestures). The crisis events leading to aggressive behavior helped us to narrow down gestures that we tried to identify as the main part of the thesis. Several research and studies were conducted on risky behavior and human emotions. However, this thesis contributed immensely towards the personalized gesture detection of the veteran population suffering from PTSD. This is a significant work done in the research field of studying risky human behavior.

The amount of quality data that we collected while doing the research is noteworthy. The data set collected are not only rich with accelerometer data from the gesture movements, it has four other physiological data (heart rate, electrodermal activity, blood volume pulse and body temperature) that are unique to any research study done so far. Although the application of clustering and classification algorithms were simple and straight forward, the findings are remarkable. Capturing accelerometer data from E4 sensor and tagging them in gesture clusters and eventually developing a predictive model that predicts the aggressive gestures is a huge contribution of the thesis. 
In summary we were able to achieve the following research goals-

- Distinctively and reliably identify a set of clusters representing aggressive gestures

- Narrow down to the smallest number of gesture that are most informative

- Pin-point labeling of the accelerometer data and gestures

- Develop a predictive model with classifier algorithms to predict risky gestures

\subsection{Storage and Accessibility of the Data Collected}

The labeled data set along with all the other physiological data are downloaded in .csv file format and stored in an online file storage repository Box. The research team has access to the data, the formulas used to synchronize the start and end time of the gestures. The video clips recorded during the data collection are stored as well for future reference. Refer to Appendix B for information how to access the data.

\subsection{Lessons Learned}

We as a research team have considerably learnt so much about the veteran population and the symptoms they show when they are suffering from PTSD. Collection of data was a big portion of the thesis. Data collection techniques and methods were developed and learned. We have done a lot of work on data pre-processing, data timestamp synchronization and labeling. These findings and techniques are a stepping stone to future researches on data analysis on wearable sensor data.

\subsection{Broader Impact}

The research outcome would directly impact the US military veterans. The predictive model could help improve the peer mentor support system existing in 
Dryhootch of America. This can be adopted as a waring trigger system by the Veteran Affairs across the nation. Individuals suffering from PTDS or anger issues can benefit from the research outcome i.e. the predictive model. The use of wearable sensors is becoming more and more popular with time. If this research finding is adopted by mass population, we would have enormous amount of data. With endless data we could train the model to come up with better classification and eventually make better prediction by improving the classification approach. This idea of gesture detection can be used in elderly care monitoring as well as in other health and nursing arena.

\subsection{Future Work}

The research is not limited to what we have done and presented here in the thesis paper. There are ways to improve the research approach. One of the important extension of the thesis could be to overcome the hardware limitation. As we know the E4 accelerometer sensor captures data form $-2 \mathrm{~g}$ to $+2 \mathrm{~g}$ range. During out research we have noticed a fair amount to data points towards the end of the range, which were eventually marked as outliers. If we can overcome the hardware limitation by extending the data capture range of the E4 device, we could have collected more data that what we have now.

Another future work for this thesis is to study non-angry gesture data. We focused only on angry gesture data. It would be interesting to see what the non-angry (normal) gesture data from the accelerometer sensor looks like. An important step would be to compare the angry gesture data with the non-angry data. This would help us to see how accurately the predictive model actually works in real world and if it could differentiate between gestures that are not based on the expression of anger or aggressive behavior. 


\section{BIBLIOGRAPHY}

[1] Borders, A., McAndrew, L. M., Quigley, K. S., \& Chandler, H. K. (2012).

Rumination moderates the associations between PTSD and depressive symptoms and risky behaviors in US veterans. Journal of Traumatic Stress, 25(5), 583-586.

[2] Reisman, M. (2016). PTSD Treatment for Veterans: What's Working, What's New, and What's Next. Pharmacy and Therapeutics, 41(10), 623-634.

[3] Puente, A. E. (2017). Re-conceptualizing Post-Traumatic Stress Disorder as a Neuropsychological Condition/اضطر اب ما بعد الصدمة كحالة نفسية_عصبية :نظرية بديلة/. Bethlehem University Journal, 34, 105-112.

[4] Akl, A., \& Valaee, S. (2010, March). Accelerometer-based gesture recognition via dynamic-time warping, affinity propagation, \& compressive sensing. In Acoustics Speech and Signal Processing (ICASSP), 2010 IEEE International Conference on (pp. 22702273). IEEE.

[5] Roggen, D., Calatroni, A., Rossi, M., Holleczek, T., Förster, K., Tröster, G., Lukowicz, P., Bannach, D., Pirkl, G., Ferscha, A. and Doppler, J., 2010, June. Collecting complex activity datasets in highly rich networked sensor environments. In Networked Sensing Systems (INSS), 2010 Seventh International Conference on (pp. 233-240). IEEE.

[6] Intille, S. S., Rondoni, J., Kukla, C., Ancona, I., \& Bao, L. (2003, April). A contextaware experience sampling tool. In CHI'03 extended abstracts on Human factors in computing systems (pp. 972-973). ACM.

[7] Atrey, P. K., Hossain, M. A., El Saddik, A., \& Kankanhalli, M. S. (2010). Multimodal fusion for multimedia analysis: a survey. Multimedia systems, 16(6), 345-379.

[8] Bernardos, A. M., Tarrío, P., \& Casar, J. R. (2008, August). A data fusion framework for context-aware mobile services. In Multisensor Fusion and Integration for Intelligent Systems, 2008. MFI 2008. IEEE International Conference on (pp. 606-613). IEEE.

[9] Siewiorek, D., Smailagic, A., Furukawa, J., Krause, A., Moraveji, N., Reiger, K., Shaffer, J. and Wong, F.L., 2003, October. Sensay: A context-aware mobile phone. In null (p. 248). IEEE. 
[10] Liu, J., Zhong, L., Wickramasuriya, J., \& Vasudevan, V. (2009). uWave: Accelerometer-based personalized gesture recognition and its applications. Pervasive and Mobile Computing, 5(6), 657-675.

[11] Dey, A. K., Sohn, T., Streng, S., \& Kodama, J. (2006, May). iCAP: Interactive prototyping of context-aware applications. In International Conference on Pervasive Computing (pp. 254-271). Springer, Berlin, Heidelberg.

[12] US Department of Veteran Affairs. (2018). PTSD: National Center for PTSD. [online] Available at: https://www.ptsd.va.gov/public/materials/apps/ [Accessed 21 May 2018].

[13] Rizia, Rizwana, "A Mobile Health Approach to Assist Veterans Reintegrating into Civilian Life" (2016). Dissertations (2009 -). Paper 633.

http://epublications.marquette.edu/dissertations_mu/633

[14] O'Brien, Casey James, "mPeer: A Mobile Health Approach to Monitoring PTSD in Veterans" (2013). Master's Theses (2009 -). Paper 231.

http://epublications.marquette.edu/theses_open/231

[15] Patwary, A B M Kowser, "mCAT: Development of a Generic mHealth Tool for Continuous Assessment, Automatic Intervention, and Analysis" (2016).Master's Theses (2009 -). Paper 351. http://epublications.marquette.edu/theses_open/351

[16] Johnson, Nadiyah Frances, "Social-Context Middleware for At-Risk Veterans" (2016). Master's Theses (2009 -). Paper 349.

http://epublications.marquette.edu/theses_open/349

[17] Dryhootch. (2018). Peer Support. [online] Available at: http://www.dryhootch.org/ [Accessed 12 Mar. 2018].

[18] Rossini, N. (2012). Reinterpreting Gesture as Language.

[19] Georgakopoulou, A. (2007). Small Stories, Interaction and Identities, John Benjamins Publishing.

[20] Franco, Z., Hooyer, K., Roushan, T., O’Brien, C., Johnson, N., Watson, B., SmithWatson, N., Tasse, J., Semaan, B., Flower, M., and Ahamed, S.I. (2018 accepted) 
Detecting \& Visualizing Crisis Events in Human Systems: an mHealth Approach with High Risk Veterans. Proceedings of the 15th Information Systems for Crisis Response and Management (ISCRAM 2018) Conference, Rochester, NY.

[21] Images taken from Online. [Available at] https://www.gonomad.com/1766-italianwithout-words-using-hand-gestures-in-conversation, http://www.alamy.com/stock-photoangry-cartoon-face-136247099.html, https://www.ikigaiway.com/2008/the-two-deadliestfists-of-karate/, https://www.shutterstock.com/image-photo/hand-gesture-arm-fistpunching-air-304199765, http://nd.zp.ua/zp/2319-zaporozhskiy-chinovnik-pomogischeznut-4-millionam-byudzhetnyh-sredstv.html, https://www.youtube.com/watch?time_continue=109\&v=dRQWEhyy6M8, http://coolspotters.com/activities/giving-the-finger

[22] Harper, M., \& Cole, P. (2012). Member checking: can benefits be gained similar to group therapy?. The Qualitative Report, 17(2), 510-517.

[23] Creswell, J. W., \& Miller, D. L. (2000). Determining validity in qualitative inquiry. Theory into practice, 39(3), 124-130.

[24] Empatica. (2018). Real-time physiological signals | E4 EDA/GSR sensor. [online] Available at: https://www.empatica.com/en-eu/research/e4/ [Accessed 6 Mar. 2018].

[25] Bureau International des Poids et Mesures (2006). "The International System of Units (SI)" (PDF) (8th ed.): 131. Retrieved 2009-11-25.

[26] "SI Unit rules and style conventions". National Institute For Standards and Technology (USA). September 2004. Retrieved 2009-11-25

[27] www.askix.com/acelerometro-y-giroscopio-tutorial_2.html

[28] Empatica. (2018). E4 for Developers. [online] Available at: https://developer.empatica.com/ [Accessed 7 Mar. 2018].

[29] Empatica Support. (2017). Session start-time format and synchronization. [online] Available at: https://support.empatica.com/hc/en-us/articles/202800715-Session-starttime-format-and-synchronization- [Accessed 4 Mar. 2018]. 
[30] The Spreadsheet Page. (2018). Excel Tips From John Walkenbach: Converting Unix Timestamps. [online] Available at:

http://spreadsheetpage.com/index.php/tip/converting_unix_timestamps/[Accessed 21 Feb. 2018].

[31] Empatica Support. (2015). How is the acceleration data formatted in Empatica Connect?. [online] Available at: https://support.empatica.com/hc/enus/articles/202028739-How-is-the-acceleration-data-formatted-in-Empatica-Connect[Accessed 21 Jan. 2018].

[32] Judd, C. M., McClelland, G. H., \& Ryan, C. S. (2011). Data analysis: A model comparison approach. Routledge.

[33] Judd, C. M., McClelland, G. H., \& Culhane, S. E. (1995). Data analysis: Continuing issues in the everyday analysis of psychological data. Annual review of psychology, 46(1), 433-465.

[34] Tukey, J. W. (1962). The future of data analysis. The annals of mathematical statistics, 33(1), 1-67.

[35] Borman, S. (2004). The expectation maximization algorithm-a short tutorial. Submitted for publication, 1-9.

[36] Talvitie, J. (2013). Expectation Maximization - Introduction to EM algorithm. [ebook] Hervanta: Tampere University of Technology. Available at: http://www.cs.tut.fi/kurssit/TLT-5906/EM_presentation_2013.pdf [Accessed 9 Jan. 2018].

[37] Bronshtein, A. (2017). A Quick Introduction to K-Nearest Neighbors Algorithm. [online] Medium. Available at: https://medium.com/@adi.bronshtein/a-quickintroduction-to-k-nearest-neighbors-algorithm-62214cea29c7 [Accessed 2 Jan. 2018].

[38] Dardas, N. H., \& Georganas, N. D. (2011). Real-time hand gesture detection and recognition using bag-of-features and support vector machine techniques. IEEE Transactions on Instrumentation and measurement, 60(11), 3592-3607.

[39] Han, J., Award, G. M., Sutherland, A., \& Wu, H. (2006, April). Automatic skin segmentation for gesture recognition combining region and support vector machine active 
learning. In Automatic Face and Gesture Recognition, 2006. FGR 2006. 7th International Conference on (pp. 237-242). IEEE.

[40] Dardas, N., Chen, Q., Georganas, N. D., \& Petriu, E. M. (2010, October). Hand gesture recognition using bag-of-features and multi-class support vector machine. In Haptic Audio-Visual Environments and Games (HAVE), 2010 IEEE International Symposium on (pp. 1-5). IEEE.

[41] Liu, Y., Gan, Z., \& Sun, Y. (2008, August). Static hand gesture recognition and its application based on support vector machines. In Software Engineering, Artificial Intelligence, Networking, and Parallel/Distributed Computing, 2008. SNPD'08. Ninth ACIS International Conference on (pp. 517-521). IEEE. 


\section{APPENDICES}

\section{APPENDIX A}

\section{Data collection frequency and unit of E4 sensor. A detailed description of the sensor} parameters

.csv files in this archive are in the following format:

The first row is the initial time of the session expressed as unix timestamp in UTC.

The second row is the sample rate expressed in $\mathrm{Hz}$.

TEMP.csv

Data from temperature sensor expressed degrees on the Celsius $\left(\hat{\mathrm{A}}^{\circ} \mathrm{C}\right)$ scale.

EDA.csv

Data from the electrodermal activity sensor expressed as microsiemens ( $\left.\hat{\mathrm{I}}^{1} / 4 \mathrm{~S}\right)$.

BVP.csv

Data from photoplethysmograph.

\section{ACC.csv}

Data from 3-axis accelerometer sensor. The accelerometer is configured to measure acceleration in the range $[-2 \mathrm{~g}, 2 \mathrm{~g}]$. Therefore, the unit in this file is $1 / 64 \mathrm{~g}$.

Data from $\mathrm{x}, \mathrm{y}$, and $\mathrm{z}$ axis are respectively in first, second, and third column. 


\section{IBI.csv}

Time between individuals heart beats extracted from the BVP signal.

No sample rate is needed for this file.

The first column is the time (respect to the initial time) of the detected inter-beat interval expressed in seconds (s).

The second column is the duration in seconds (s) of the detected inter-beat interval (i.e., the distance in seconds from the previous beat).

HR.csv

Average heart rate extracted from the BVP signal.The first row is the initial time of the session expressed as unix timestamp in UTC.

The second row is the sample rate expressed in $\mathrm{Hz}$.

tags.csv

Event mark times.

Each row corresponds to a physical button press on the device; the same time as the status LED is first illuminated.

The time is expressed as a unix timestamp in UTC and it is synchronized with initial time of the session indicated in the related data files from the corresponding session. 


\section{APPENDIX B}

\section{Access to the Research Data}

If anyone is interest to get further information regarding this thesis research, and need access to the data we collected along with the data pre-processing steps, please refer to the link below-

Box: Secure File Sharing, Storage, and Collaboration https://ubicomplab.app.box.com/folder/46847025768

For any additional information, please contact the author or send an e-mail to ubicomp2@gmail.com 